\title{
The Rules of Pulsatile Neurostimulation
}

\author{
Cynthia R. Steinhardt ${ }^{1}$, Diana E. Mitchell ${ }^{2}$, Kathleen E. Cullen ${ }^{1,3,4}$, Gene Y. Fridman ${ }^{1,3,5}$
}

1. Department of Biomedical Engineering, Johns Hopkins School of Medicine, Baltimore, MD, USA

2. Department of Neurosciences, Faculty of Medicine, University of Montreal, Montreal, QC, Canada.

3. Department of Otolaryngology-Head and Neck Surgery, Johns Hopkins School of Medicine, Baltimore, MD, USA

4. Department of Neuroscience, Johns Hopkins School of Medicine, Baltimore, MD, USA

5. Department of Electrical and Computer Engineering, Johns Hopkins University, Baltimore, MD, USA

\section{Abstract}

Electrical stimulation of neural responses is used both scientifically in brain mapping studies and in many clinical applications such as cochlear, vestibular, and retinal implants. Due to safety considerations, stimulation of the nervous system is restricted to short biphasic pulses. Despite decades of research and development, neural implants are far from optimal in their ability to restore function and lead to varying improvements in patients. In this study, we provide an explanation for how pulsatile stimulation affects individual neurons and therefore leads to variability in restoration of neural responses. The explanation is grounded in the physiological response of channels in the axon and represented with mathematical rules that predict firing rate as a function of pulse rate, pulse amplitude, and spontaneous activity. We validate these rules by showing that they predict recorded vestibular afferent responses in macaques and discuss their implications for designing clinical stimulation paradigms and electrical stimulation-based experiments.

\section{Introduction}

Electrical stimulation is ubiquitous in modern treatments for neurological disorders ${ }^{1}$ and studies of neural function. ${ }^{2,3}$ It has a longstanding and intertwined history with the field of neuroscience, starting from the first studies showing neurons could be activated by electrical stimulation. ${ }^{4,5}$ In the present, electrical microstimulation remains a prominent way to understand neural function and connectivity at local and interregional levels of the nervous system. ${ }^{3,6,7}$ Clinically, it is not only relied on for brain mapping to inform treatments; ${ }^{6}$ usages have expanded to neural prostheses for restoration of perception ${ }^{1,8}$ and treating neuropsychiatric disorders ${ }^{9}$. The main driver of safety standards has been developing paradigms suitable for long-term, implantable devices, which led to the restriction of current delivery to biphasic, charged-balanced pulses that prevent electrochemical reactions at the electrode interface and neural damage. ${ }^{8}$ Resultantly, electrical stimulation has become synonymous with this form of pulsatile stimulation across applications. Implantable pulse generators have been used in neural implants since the 1950s to treat impairments including hearing ${ }^{10}$ visual, $^{11}$ and balance ${ }^{12}$ and movement disorders ${ }^{13}$. These devices use changes in pulse rate and amplitude to modulate local neural activity to replace natural function. While the list of clinically viable prostheses based on this technology is rapidly growing, their ability to restore encoding of the full range of information remains limited. ${ }^{1}$ Even in the cochlear implant, which has been continually improved since the initial introduction in $1957,{ }^{14}$ speech perception is restored, but pitch perception, which is encoded in finetiming of firing rates, is at a deficit, likely leading to lack of music appreciation and tonal language perception. ${ }^{14,15}$ Many explanations have been proposed for limitations in restored function for the each sensory prostheses, such as noise reduction in the front-end processing in cochlear implants ${ }^{16}$, pathological neural 
bioRxiv preprint doi: https://doi.org/10.1101/2021.08.18.456731; this version posted August 19, 2021. The copyright holder for this preprint (which was not certified by peer review) is the author/funder, who has granted bioRxiv a license to display the preprint in perpetuity. It is made available under aCC-BY-NC-ND 4.0 International license.

rewiring of the retina ${ }^{17}$, or central nervous system adaptation to vestibular stimulation 18 . These findings imply that present stimulation paradigms do not restore the natural local responses and, in turn, raise significant questions about the validity of using either microstimulation ${ }^{2,6,19}$ or cortico-cortical evoked responses to understand natural local and interregional connectivity and function. ${ }^{3,7}$

Here we argue that a common element associated with the inability to optimize the clinical systems may be the complexity and unpredictability of evoked neural activity in response to stimulation pulse trains. While substantial experimental evidence exists for pulsatile effect on neurons, a systematic theory of how pulse rate and pulse amplitude affect neurons of varying spontaneous activity has not been established. What is established, however, is that pulses produce complex effects at the axon ${ }^{19-21}$ (e.g., facilitation and blocking of future pulses), and spontaneous activity significantly impacts the induced firing rate in response to pulses. ${ }^{22}$ In this study, we perform a systematic investigation of the relationship between pulse rate ( $p r)$, pulse amplitude $(I)$, and spontaneous rate $(S)$ in a biophysical model of a vestibular afferent, where baseline spontaneous firing has been shown to impact pulsatile stimulation. ${ }^{22}$ We use a computational model to determine the physiological underpinnings these non-linear effects on firing rate and describe them with equations related to measurable and controllable quantities: $f r=\varphi(p r, l, S)$. We further validate this framework with experimental observations of vestibular afferents obtained in non-human primates in response to vestibular canal stimulation with pulse trains of different amplitudes and rates. We determine whether these non-linearities can be corrected for algorithmically in neural implants and conclude by discussing their implications for interpreting electrical stimulation studies.

\section{Results}

To investigate the relationship between pulse parameters and induced firing rate $(f r)$, we recorded neuronal activity from vestibular afferents of the semicircular canal in awake behaving primates receiving pulse train stimuli from 25 to 300 pps with amplitudes between 0 to $240 \mu \mathrm{A}(0-100 \%$ of the safe range) (Fig. 1a, b; See Methods). For a typical afferent with spontaneous rate of $40 \mathrm{sps}$ (Fig. 1c left), fr is sensitive to the pulse amplitude $(I)$ and pulse rate (pr) of a pulse train. Suprathreshold pulses are thought to produce an action potential (AP) within milliseconds of pulse delivery, regardless of spontaneous activity, yielding a relationship between $p r$ and $f r$ that would increase with slope of $1 \mathrm{sps} / \mathrm{pps}$ independent of $S$ (Fig. 1c left red line). We refer to this as a linear relationship in the paper and anything to the contrary as non-linear. Such relationship was not observed in this or any recorded afferent for any stimulation amplitude. Instead, slopes ranged from -0.2 to $0.5 \mathrm{sps} / \mathrm{pps}$. This suggests that a pulse produces less than one spike over a trial, can suppress activity (negative slope), and that the responses are highly variable.

We determined the factors that play into this variability by simulating pulsatile stimulation of a single vestibular afferent using a modified biophysical model developed by Hight and Kalluri ${ }^{23}$ (Fig. 1d, See Methods). ${ }^{24}$ To validate the model, we tuned it to replicate a typical afferent and simulate the primate experiments (Fig. $1 \mathrm{~b}$ ). The simulation, shown for a single amplitude in Figure 1e, closely replicated experimental results - including location and directionality of the bends (arrows) in the pulse rate-firing rate relationship (PFR) ( $N=50, r m s=$ $11.4 \pm 4.6 \mathrm{sps}$ ). We then conducted a full sweep of pulse amplitudes (I from 0 to $300 \mu \mathrm{A}$ ) for the same pulse rates, revealing a broad range of non-linear additive and blocking effects that depend on / (Fig. 1f left). The bends in the PFR are observed for all amplitudes at the same pulse rate (Fig. $1 \mathrm{f}$ right). Both findings contradict standard assumptions of an activation threshold and linear PFR.

We expanded on this simulation to identify the relationship between pulse parameters (pulse amplitude, pulse rate, pulse timing) and spontaneous activity (rate, regularity, conductance) and the observed complexities in the PFR. A complete set of responses was generated at finer sampling of combinations of each parameter (Fig. 2). We found that the primary determiners of the induced firing rate are the pulse amplitude and the spontaneous rate of a neuron. In Fig. 2, the PFR is shown in different panels as amplitude increases. The PFR 
with no excitatory post-synaptic currents (EPSCs) and therefore no spontaneous firing in the model (Fig. 2,black) shows the same non-linearities observed with spontaneous activity, implying pulses create these effects (Fig. 1). Within panels, the effect of $S$ is shown for the same $/$ and sampling of $p r$. Increasing spontaneous rates are shown in colors from light green (5 sps) to dark blue (131.8 sps). The same nonlinearities are present as when there is no spontaneous activity (black). Spontaneous activity appears to primarily change the responsiveness of the axon to pulses, with higher $S$ leading to smaller changes in firing rate.

The rest of our investigation is devoted to analyzing the physiological reasons for the full range of phenomena seen in Figure 2. A smooth change in phenomena with increased pulse amplitude that can be broken into fundament rules and approximated with mathematical equations. The equations are based on the underlying biophysical phenomena but represented with time-invariant equations with respect to pulse parameters and spontaneous activity. By analogy temperature of an object can be described in terms of individual atomic collisions, but a more useful description would be in terms of mass of an object and its thermal conductivity. Because the rules are principally dependent on pulse amplitude, their relevance is shown by the thickness of color-coded lines below the panels that are labeled on the right. For example, Suppression of Future Pulses (SFP), shown with a yellow line for amplitudes over $252 \mu \mathrm{A}$, is prevalent only for high amplitude pulses. The rules we developed successful capture these effects. In Figure 2 and all subsequent figures, the PFR prediction is plotted with thin red traces on top of the corresponding computational model outputs.

\section{Effects of Pulses on Neurons with No Spontaneous Activity}

To isolate the contributions of pulses to the observed non-linearities in PFR, we first investigated the effects of pulses in the absence of spontaneous activity (Fig. 2 black traces). The prevalent feature of these traces is bends that occur at specific pulse rates (Fig. 2 rows 2-3).

In Figure 3a, we examine these bends in more detail as a function of amplitude. The PFR starts with slope fr=pr (i.e. one spike for each pulse) and transition from the line $f r=p r /(N-1)$ to $f r=p r / N$ where $N=2,3,4, \ldots . . ;$ t these transitions occur at evenly spaced pulse rates. The physiological reason for this effect is that delivering a pulse, evokes an AP, but then creates an afterhyperpolarization that prevents the axon for a time $t_{b}$ from evoking an AP in response to a subsequent pulse. As the $p r$ increases, the inter-pulse-interval $(\rho)$ decreases and more pulses fall into this blocked range indicated in blue shaded area in Figure 3a, right. Meanwhile, the block window $\left(t_{b}\right)$ changes only as a function of $I$.

$t_{b}$ however describes only part of the pulse-pulse interaction dynamics. During this time, a pulse will not produce a spike, or equivalently, the probability of evoking a spike, $P_{A P}=0$ (Fig. 3a, b blue). After this initial time window, the probability of pulses being blocked from initiating spikes decreases as channel states slowly recover for time $t_{p b}(I)$ (Fig. 3b, d). In this partial elimination (PE) window, $P_{A P}$ increases gradually until it reaches 1 at $t_{p b}(I)$, after which a subsequent pulse will evoke an AP.

These effects are combined into the Pulse-Pulse Block (PPB) rule:

$$
f r=\frac{\left(\frac{1}{\rho}\right)}{\operatorname{ceil}\left(\frac{t_{b}(I)}{\rho}\right)+P E(I, \rho)}
$$

$t_{b}(I)$ is a fixed value for a given $I$. The ceil() term enforces the division by $N$ in $f r=p r / N$ when $\rho$ takes values $\frac{t_{b}(I)}{N}>\rho>\frac{t_{b}(I)}{N-1}$. At low amplitudes, $t_{b}(I)$ is long due to the small changes in the membrane potential being propagated by the channel dynamics such that it will block even multiple pulses. As / increases, the cathodal 
phase of pulses drives the axon to threshold more easily, leading to minimum $t_{b}(I)$ around $150 \mu \mathrm{A}$. At high $I$, delivering a pulse is more likely to produce a cathodal block, causing $t_{b}(I)$ to increase again. $P E(I, \rho)$ is an increase in probability of a pulse being blocked as $\rho$ decreases and the pulse occurs closer to $t_{b}(I) / N$ (Fig. $3 \mathrm{~b}-\mathrm{c}$ red).

The contribution of these rules to the PFR can be seen in Figure 3c. As pulse rate increases for a given amplitude, a larger range of pulse rates fall into the PE zone due to smaller inter-pulse-intervals (Fig. 3c Effect 1). We also note that, as I increases, recovery take more time, leading to longer PE zone (Fig. $3 c$ Effect 2, 3d, Methods Eq. 5-6).

At high I, we see new effects: Suppression of Future Pulses (SFP, yellow) and Pulse Dynamic Loop (PDL, tan) (Fig. 3e). PDL is caused by pulses being of the right size and timing to create an extended competition between the $\mathrm{K}$ and $\mathrm{Na}$ channels dynamics that prevents AP production. SFP is a more severe form of this effect. The sodium h-gate is driven to a state that it cannot recover from in time to respond to the next pulse presentation, keeping the channel inactivated and preventing all following pulses from inducing APs (Fig. 3f). In sum, pulses produce additive and blocking effects in the absence of spontaneous activity, and these effects on the PFR can be reproduced with Eq. 1, an equation that depends on I and pr (Fig. 3c,e-f, Methods Eq. 11-13).

\section{Effects of Pulses on Spontaneously Firing Neurons}

When spontaneous activity was introduced into the model, there were additional pulse-spontaneous interactions that were separable from the pulse-pulse interactions described above. At different pulse rates, spontaneous APs (green) and pulse-induced APs (black) vary in ratio of one to another (Fig. 4a) shown for $30 \mathrm{~Hz}$ and $80 \mathrm{~Hz}$ in the example. The underlying mechanism is an extended perturbation of axonal channel dynamics in the time after a pulse-induced or spontaneous AP which results in an additive or blocking effect on the following EPSC or pulse. The extent and directionality of these interactions depends on pulse amplitude and scales differently with / than PPB.

Fortunately, although spontaneous activity is stochastic, timing of spontaneous APs is uncorrelated to pulse timing and approximately uniformly distributed between pulses (Fig. 4b), affecting all pulse-evoked APs equally independent of pulse rate. The probability that a pulse produces an AP after a spontaneous AP, $p_{s p}$ increases only as a function of $I$, because, as I increases, pulses become large enough to break through the aftereffects of spontaneous APs; the probability of an EPSC producing an AP after a pulse, $p_{p s,}$ depends on pulse amplitude I and pulse rate $p r$ (Methods Eq. 7-9). As pr increases, the time between pulses is smaller but $t_{p s,}$ the time after a pulse in which pulses interact with spontaneous APs remains the same (Fig. $4 \mathrm{~b}$ left). Therefore, the portion of effected spontaneous spikes increases linearly with $p r$ until $p_{p s}=1$, meaning that all spontaneous APs within the inter-pulse window are affected (Fig. $4 \mathrm{~b}$ right). $p_{p s}$ and $p_{s p}$ vary together, producing additive or blocking effects depending on I (Fig. 4c-h).

With no spontaneous activity at low pulse amplitude, pulses self-facilitate, producing APs at high pulse rates (Fig. 4c black). At low pulse amplitudes, Pulse-Spontaneous Addition (PSA) occurs when spontaneous activity adds to sub-threshold pulse-induced activity facilitating APs even at low pulse rates (Fig. 4c,e turquoise). This effect additionally smooths the pulse-induced changes at high amplitudes and eliminates the PDL effects more as spontaneous rate increases(Fig. 4d). At high pulse amplitudes, pulses begin to self-block due to the SFP rule described earlier. At these same amplitudes, the pulse-spontaneous spike interactions produce PulseSpontaneous Block (PSB) and block each other with the same probability as PSA (Figure 3e,f). 
At intermediate pulse amplitudes, when pulses are great enough to produce APs, Spontaneous Pulse-Block (SPB) occurs (Fig. 4g). Pulse-evoked APs that would be produced with no spontaneous activity are blocked. For $p r>S, S$ pulses are blocked, so $\max (\Delta \mathrm{fr})$ reduces to $\Delta \mathrm{fr}_{\mathrm{S}=0}-\mathrm{S}$ (Fig. $4 \mathrm{~g}, \mathrm{~h}$ ) but the slope is one until the first bend. For $p r<S$, we observe a slope less than one, because aftereffects of spontaneous activity can block multiple pulses, but pulses self-facilitate between spontaneous APs when $p r>S$. This slope increases with I until I is large enough that additive effects switch to blocking effects. Together, these effects show spontaneous activity has a significant impact on the maximum inducible firing rate. These effects are also incorporated in the prediction equation: $f r=\varphi(p r, I, S)$ (See Methods).

We parameterized our equation for how the rules change with $p r, l$, and $S$ to the simulations, resulting in accurate predictions across all conditions ( $\max r m s=4.0 \pm 0.41 \mathrm{sps}$, Supplemental Table 1) (Fig. 2 red). We can then interpolate the change in parameters with $I$ and $S$ and predict probability of pulse-pulse interactions, pulse-spontaneous interactions, and induce firing rate for any combination of $p r, I$, and S (See Methods).

\section{Predictability of Single Neuron Responses}

We tested the ability of the equations to predict neural firing responses to pulse trains recorded at different pulse amplitudes in macaque vestibular afferents. Because the distance and tissue conductance at each recorded neuron affect the current amplitude experienced at the axon, we do not expect a one-to-one mapping between the predicted pulse amplitude $\left(I_{\text {pred }}\right)$ and experimentally delivered pulse amplitude $\left(I_{\text {expt }}\right)$. We determined whether this mapping is accurate by finding the $I_{\text {pred }}$ that minimizes the rms error between the predicted firing rate at every pulse rate given the measured spontaneous activity and the experimentally recorded pr-fr values(See Methods). Under these conditions, the equations closely match the experimentally obtained firing rates (Table 1, Fig. 5a-b). The different neurons map to different ranges of the simulated pulse amplitudes. There is evidence of facilitation/PSA (Afferent \#3), mid-range additive and blocking effects/PPB/SPB (Afferent \#1,\#4), and suppression effects/SFP/PSB (Afferent \#2), providing support for each rule (Fig. 5a). Comparing Afferent \#1 and \#2 reveals another complexity. Both neurons receive the same $p r$ and $I$ and have similar $S$ but respond at different parts of the mapping (Fig. 5b). One explanation is that vestibular afferents have a large variance in conductances. ${ }^{23}$ Simulations within biophysical bounds suggest that reduced membrane conductances of neurons leads to more rapid transition in the mapping in Fig. 2 from low I to high I responses with current increase (Supplemental Fig. 1), further explaining the $l_{\text {expt }}-I_{\text {pred }}$ relationship for Afferents \#2 and \#3.

Table 1. RMS Values and Percent Error Per Afferent

\begin{tabular}{|c|c|c|c|c|c|}
\hline \multirow{2}{*}{ Afferent } & \multicolumn{5}{|c|}{ RMS Value (sps) [\%Error] } \\
\hline \multirow{2}{*}{$\mathbf{1}$} & $\boldsymbol{I}$ (25\%) & $\boldsymbol{I}$ (50\%) & $\boldsymbol{I}$ (75\%) & $\boldsymbol{I}$ (87.5\%) & I (100\%) \\
\hline \multirow{2}{*}{$\mathbf{2}$} & 3.060 & 2.755 & 15.281 & 2.894 & 1.787 \\
& {$[1.44]$} & {$[0.93]$} & {$[3.32]$} & {$[1.96]$} & {$[1.15]$} \\
\hline \multirow{2}{*}{3} & 12.564 & 4.104 & 6.804 & 9.237 & \\
& {$[4.96]$} & {$[2.05)$} & {$[2.69]$} & {$[2.57]$} & \\
& 3.985 & 6.436 & 5.184 & 7.877 & \\
& {$[4.24]$} & {$[7.36]$} & {$[2.76]$} & {$[9.50]$} & \\
\hline
\end{tabular}



available under aCC-BY-NC-ND 4.0 International license.

\begin{tabular}{|l|l|l|l|l|l|}
\hline 4 & 1.787 & 2.452 & 15.843 & 14.881 & 4.274 \\
& {$[1.16]$} & {$[1.13]$} & {$[5.78]$} & {$[3.05]$} & {$[3.11]$} \\
\hline
\end{tabular}

\section{Implications for Current Usages of Pulsatile Stimulation}

The rules indicate pulses produce a variety of population responses even in neurons directly under an electrode responding to a stimulus of a fixed pulse rate and amplitude. A pulse delivered from an implanted electrode produces an electric field that decays with distance from an electrode. Therefore, underneath a single electrode, distal neurons will experience lower stimulation currents and would respond as if they were stimulated at a lower amplitude in Figure 2. Additionally, spontaneous activity significantly impacts the magnitude and even directionality of $\Delta f r$ for the same stimulation amplitude, producing large additive effects for low spontaneous rates ( $S<30$ sps) and large blocking effects for high spontaneous rate $(S=131$ sps) (Fig. 5c). Finally, neurons with different membrane conductances respond differently to the same stimulus. Between these effects, neighboring neurons may not respond in with the same magnitude or directionality to the same stimulus.

We additionally considered whether the rules apply under realistic clinical implant scenarios that modulate pulse rates or amplitudes, and when pulses are delivered to neurons of varying spontaneous firing regularity. A potential concern would be the effect of regularity of spontaneous spike timing compared to pulse timing. For pulse rates greater than the spontaneous rate, regularity did not affect induced firing pattern significantly (Fig. $5 d$ ). The largest differences are that the pulse elimination zone is significantly shorter and pulse rates below $S$ produce no additional pulses or block spontaneous activity (Supplemental Fig. 2). Another question is how regularity of pulse timing affects the firing rate. Even pulses programmed with ideal regularity show some jitter in delivery due to imperfect implementation of both hardware and software. We introduced 1 and 2 ms of jitter around pulse delivery times, finding the same rules apply. The jitters extend the smoothing of the $P E$, similarly to spontaneous activity, but maintain the underlying mechanism and frequency of pulse-spike interaction (Fig. 5e).

We assessed whether the rules hold when applied in the context of pulse rate (PRM) and pulse amplitude modulation (PAM) rather than pulse trains, two standard paradigms in which pulse rates change over time. We simulated sinusoidal PRM (modulation of $p r$ with fixed $/$ ) and PAM (modulation of / with fixed $p r$ ). The mapping from fixed pulse simulations (Fig. 2) predicted ranges of non-linear and linear PFR (Fig. $5 f$ grey panels) for PRM and PAM. PRM (red) and PAM (blue) show firing rates inconsistent with sinusoidal modulation (black), as predicted by our rules (Fig. 5f). Additionally, even at the $I$ of maximum responsiveness, $150 \mu \mathrm{A}$, there is a less than one-to-one response. We test how this would affect prosthetic outcome by applying a common stimulation strategy: we simulated pulse delivery at pulse rate equivalent to nature firing rate in response to a detected head velocity. ${ }^{12,25}$ When we map the corresponding pulse rate to firing rate of the afferent, the resulting firing is severely attenuated and nonlinear as shown in blue, which could account for asymmetries in vestibular ocular reflex behavior assays and reduced sensitivity to head velocity in behavioral studies and human applications (Fig. 5g). ${ }^{12,25-28,30}$

\section{Discussion}

In this study, simulations of vestibular afferents revealed a number of non-linearities in the relationship between pulse parameters and induced firing rate that are not accounted for in our uses of electrical stimulation in scientific investigations or the clinic (Fig. 2). These non-linearities have been observed before across systems: the bend in PFR (row 3 ) $^{31}$ and high frequency facilitation (row 1 ) have been observed in cochlear afferents; ${ }^{32}$ high amplitude block is observed in the sciatic nerve (row 4), ${ }^{33}$ amplitude-dependent growth of firing rates has been observed in the auditory nerve (Fig 2. row 1 ) ${ }_{i}^{34}$ our experiments in the 
bioRxiv preprint doi: https://doi.org/10.1101/2021.08.18.456731; this version posted August 19, 2021. The copyright holder for this preprint (which was not certified by peer review) is the author/funder, who has granted bioRxiv a license to display the preprint in perpetuity. It is made available under aCC-BY-NC-ND 4.0 International license.

vestibular system ${ }^{35}$ and hippocampal responses demonstrate pulse-spontaneous blocking effects (Fig. 4). ${ }^{36}$ However, each has been treated as a separate case and solutions have been attempted respective to the system.

We find that the unnatural perturbation of the axonal membrane potential by biphasic pulsatile stimulation induces these effects by creating long-term loops in the dynamics of sodium and potassium channels. Given most neurons rely on these channels or channels with similar dynamics to produce action potentials, we expect pulses induce these same non-linearities in all neurons (as supported by the cases above): bends in the PFR, facilitation, and block (Fig. 2). However, the pulse rates at which the first bend occurs ( $t_{b}$ ) or the length of suppression effects will differ depending on the time constants of the channels specific to the axon.

These findings have several important implications for electrical stimulation-based interactions with the brain. Suprathreshold pulse amplitudes (e.g. $100 \mu \mathrm{A}$ ) are assumed to drive one AP per pulse and lead to increased activation as amplitude increases by recruiting more neurons. Instead, we find individual neurons are highly sensitive to pulse amplitude because it determines the perturbation of channel state and therefore length of induced dynamic loops. Thus, neurons closer to the electrode center could produce significantly different firing rates than more distal neurons within the current field. Additionally, spontaneous activity interacts with pulses, creating additive and blocking effects by priming or deactivating channels concurrent with pulse delivery. Although spontaneous activity is stochastic and channel dynamics are non-linear, the probability of interaction can be estimated with respect to pulse amplitude, pulse rate, and spontaneous rate, because of the approximately regular timing of pulses, continual EPSC activity, and orbits in channel state. We thus created equations describing these interactions. These equations were formulated to describe each of the pulseinduced effects (PPB,PDL,SFP) and pulse-spontaneous interactions (PSA,SPB,PSB); equations that captured these main effects predicted responses of real neurons to pulsatile stimulation of varying amplitudes (Fig. $4 a$, Methods). We also found that these effects persist with jitter in pulse delivery or when there is irregularity in firing, and they predictably distort the induced firing rate during pulse rate and amplitude modulation (Fig. 5). The original intent was to develop equations that could be used to correct neural implant algorithms for nonlinear effects. However, we also uncovered a scientifically important caveat. Neighboring neurons do not typically share the same spontaneous rate. Additionally, differences in channel densities (Supplemental Fig. 2), or local tissue properties can lead to additive and blocking effects in proximal neurons. This result, which is supported by past microstimulation studies ${ }^{19,36}$, implies the population responses are not predictable without characterizations of the individual neurons, which is an intractable solution. This result has two scientific implications. First, it calls into question whether electrical stimulation-based mapping studies unveil natural effective connectivity and therefore function. Second, despite these seemingly incoherent changes in population firing, partial encoding of information occurs (e.g. restoration of hearing without pitch perception). Increases in pulse rate or pulse amplitude cause an increase in the local population response that is correctly interpreted by higher-level cortex (e.g. increased sound loudness). These findings support theories of population-level spatio-temporal integration of encoding ${ }^{37}$ and transmission ${ }^{38,39}$ of information and indicate that pulsatile stimulation could drive these functions, if delivered with the correct parameters.

Future work requires understanding the spatial distribution and proportion of excitatory and inhibitory responses to pulses. Once the spatial distribution and specificity of these responses is better understood, we can build theories around how responses to pulsatile stimulation can be coherently integrated into behavior.

Several potential solutions for neural implants also emerge. To increase controllability, a potential solution would be to eliminate spontaneous activity or inputs from other areas, for example, using gentamicin on vestibular hair cells ${ }^{40,41}$, or site-specific channel blockers in cortex. This avoids three of the uncovered non-linear effects (PSA,SPB,PSB). Additionally, using a channel dynamics perspective, a novel stimulation waveform could be designed to take advantage of dynamic loops. For example, our results show that perturbation cathodiconly pulses push the channels into an unstable state. A typical anodic recovery-phase can affect the duration of 
the evoked spike afterhyperpolarization. The shape of the recovery-phase of a pulse could be designed to keep the channels sensitized, so that when the next pulse is delivered one-to-one AP induction occurs.

Using a similar investigative approach, we uncovered the mechanisms of direct current galvanic stimulation; ${ }^{24,42-47}$ we found that, because galvanic stimulation directly modulates the axonal membrane potential, it smoothly increases and decreases firing rate, regardless of axon properties, spontaneous activity, or distant from the stimulation site. As such, galvanic stimulation overcomes the barriers to neural control uncovered in this paper. Investigating effects on channel dynamics could be informative for understanding the benefits of other stimulation paradigms.

This paper provides a coherent explanation to the variability in neural responses to pulsatile stimulation. Through the explanations posited here, we find a potential solution to a number of issues with neural control and suggest ways forward for using pulsatile and alternative forms of electrical stimulation in treatment and scientific investigations. 


\section{Figures}

a
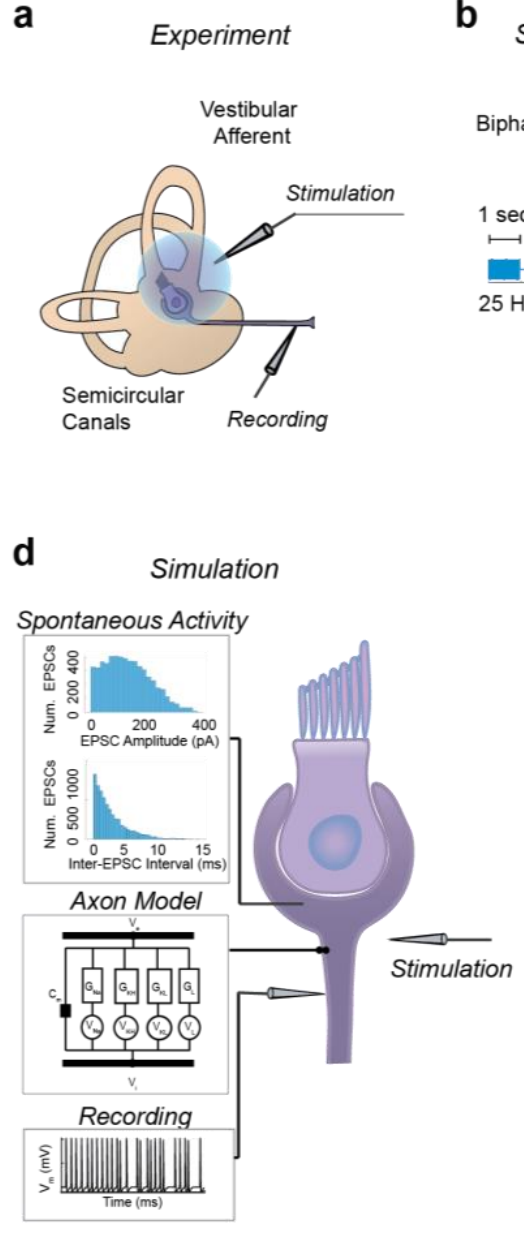

b Stimulation Paradigm

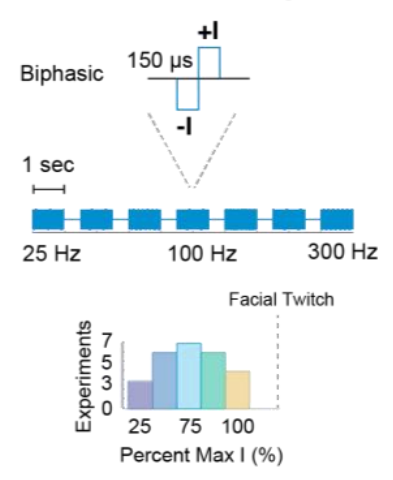

e
C

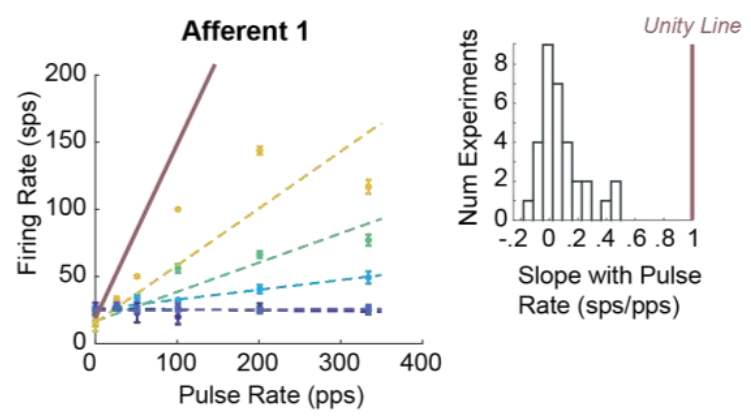

f
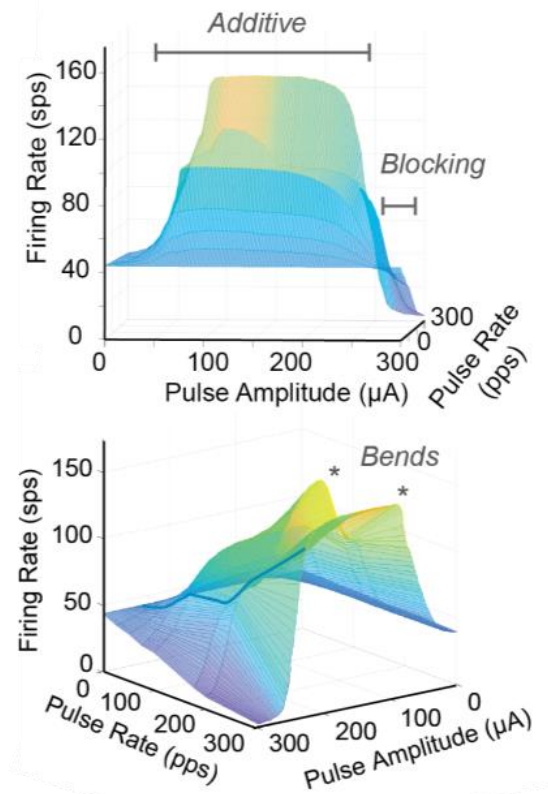

Figure 1. Paradigm for Studying Pulsatile Stimulation. a Stimulation experiments were performed on vestibular afferent in horizontal canal of macaque monkeys. b Afferents were stimulated with 1 second of fixed rate biphasic charge-balanced pulses (with $150 \mu$ s stimulation phase) from 25-300 pps. Amplitudes from 25\% to 100\% of the amplitude of facial twitch were used across subjects. c Example afferent recorded at amplitudes colored from low (purple) to high (yellow), showing non-linear change in firing rate with pulse rate. Histogram of average slope of linear-best fit across all afferents and amplitudes compared to expected unity slope: $\Delta \mathrm{fr}=1^{*} p r$. $\mathbf{d}$ A biophysical model of a vestibular afferent with realistic channel conductances was parameterized to replicate one of the irregular afferents with spontaneous rate of $42 \mathrm{sps}$. e Original data (black) compared to simulation across 50 random seeds (blue). The simulation shows evidence of nonlinearities observed in the original data (blue boxes). $\mathbf{f}$ Surface of relation between pulse amplitude, pulse rate and induced firing rate in simulated neuron based on 1 second trials. 
Change of Effects with Current Amplitude
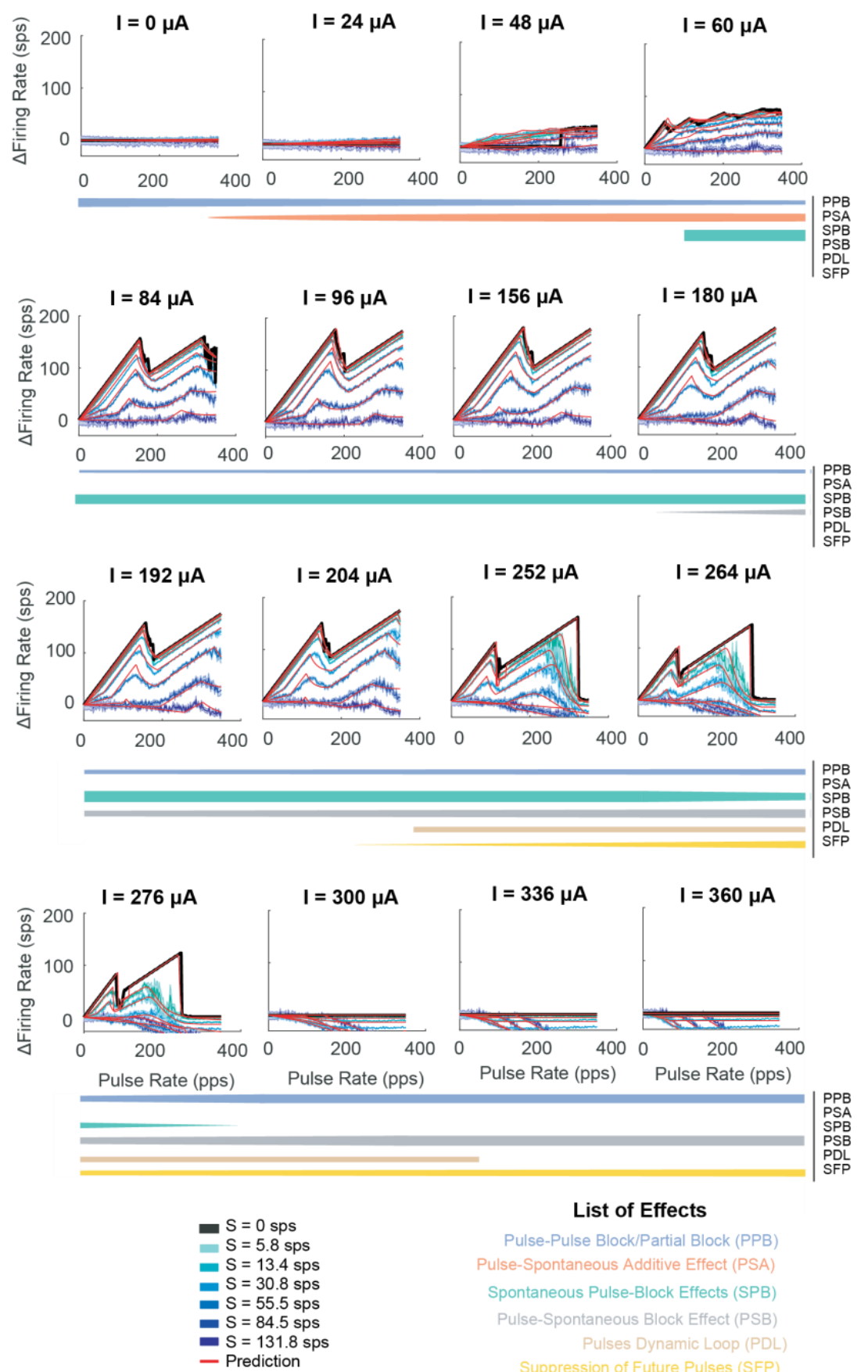

List of Effects

Pulse-Pulse Block/Partial Block (PPB)

Pulse-Spontaneous Additive Effect (PSA)

Spontaneous Pulse-Block Effects (SPB)

Pulse-Spontaneous Block Effect (PSB)

Pulses Dynamic Loop (PDL)

Suppression of Future Pulses (SFP)

Figure 2. Map of firing rate changes in response to pulse rate train presentations given spontaneous activity $S$ and pulse amplitude I. The simulation was performed at each spontaneous rate (blue-green map) and current amplitude. The change in firing rate (from the spontaneous rate) is shown with pulse rate to keep induced firing rates on the same scale. The prediction using the parameterized equations is shown for comparison (red). On the right the change in effect magnitude is shown color-coated by effect. Each row shows a transition from one effect to the next. The effects prevalent for a given range of amplitudes are shown in the left column. PPB: Pulse-pulse block/partial block, PSA: pulse-spontaneous addition, SPB: spontaneous-pulse block, PSB: pulse-

spontaneous block, PDL: pulses dynamic loop, SFP: suppression of future pulses. Nonzero spontaneous rates were generated by selecting average EPSC rates from $0.25 \mathrm{~ms}$ ( $131 \mathrm{sps}$, dark blue) to $8 \mathrm{~ms}$ ( $5 \mathrm{sps}$, light green). 
a

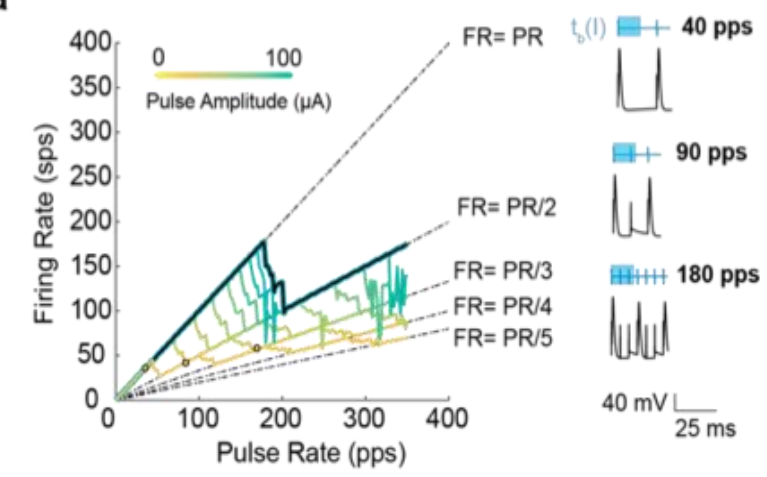

C

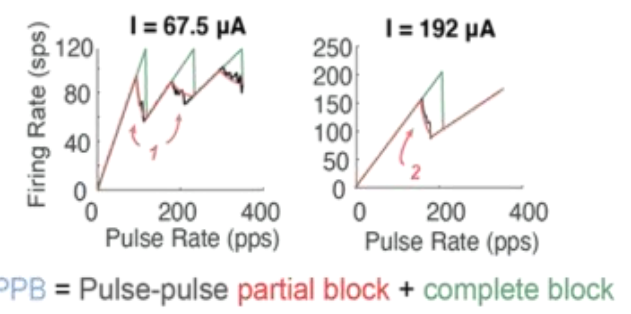

b

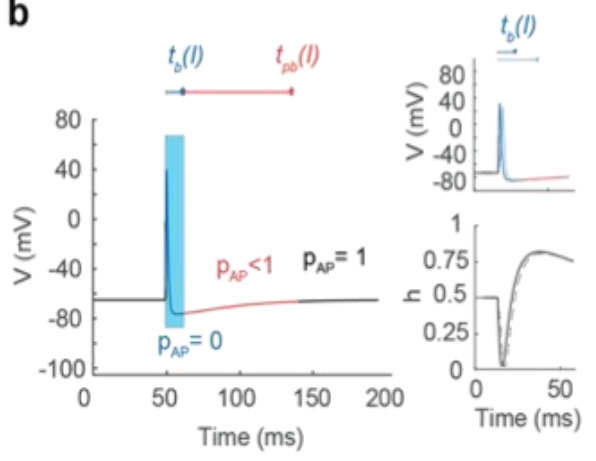

e

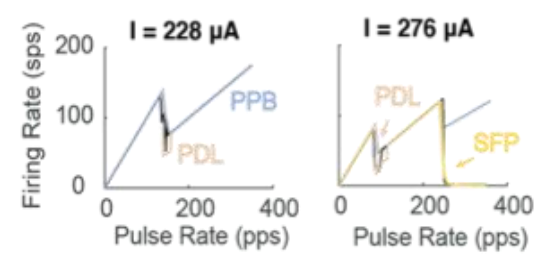

f

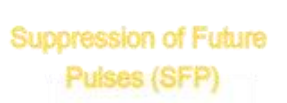

d

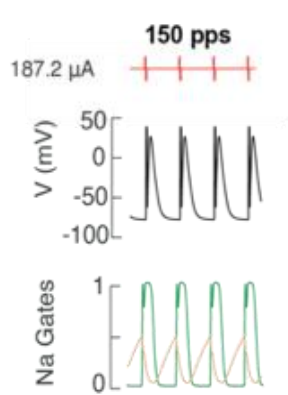

no block

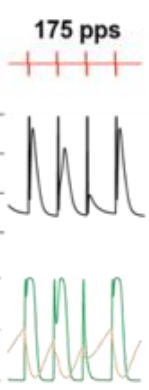

partial block
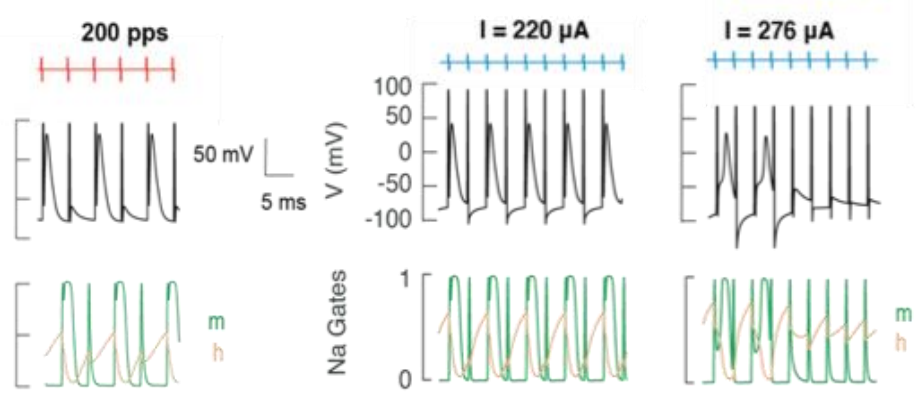

complete block

Figure 3. Effects of Pulse on a Silent Neuron. a. The length of the block zone tb changes with $I$. For one $I$, this block window leads to the firing rate reducing to subharmonics of the pulse rate depending on the ratio of the inter-pulse-interval and $t_{b}$. This effect is shown for $I=60 \mu \mathrm{A}$ (yellow dots). b. In the afterpulse window pulses change axon channel states fully blocking pulses up to tb $\mathrm{ms}$ after the pulse (blue). In the recovery period, there is a Partial Elimination (PE) zone where a subsequent pulse would be blocked with a probability that decrease to zero if pulses are $t_{\mathrm{pb}} \mathrm{ms}$ after the previous pulse. As I increases from threshold levels (dash) to higher $l$, sodium dynamics $(h)$ reach states for firing more quickly, changing the length of the block and PE zone. c. The block window (blue) and the block and partial block window (red) described by equations compared to simulated relationship (black) (see Methods). 1. PE window decreases with subharmonic jump. 2. PE window increases with $I$. d. The input pulses (red), voltage trace (black), and dynamics of the sodium channel $\mathrm{m}$-gate (green) and h-gate (yellow) that drive the PPB and SFP effect. e. At high $I$, The jump from FR=PR to FR=PR/2 creates a Pulse Dynamic Loop (PDL) that causes $1 / 3$ or less of pulses to produce APs until the pulse rate increases, changing the dynamic paper so $F R=P R / 2$ (left). A Suppression effect occurs at pulse rates that would at lower $/$ produce $F R=P R / 2$ leading to $F R=0$ (right). f. The input pulses (blue), voltage trace (black), and dynamics of the sodium channel m-gate (green) and h-gate (yellow) that drive the PPB and SFP effect. 

available under aCC-BY-NC-ND 4.0 International license.

a
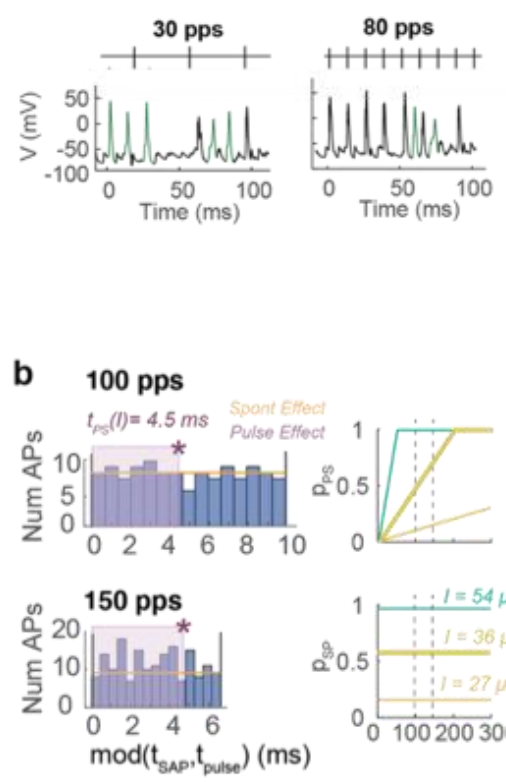

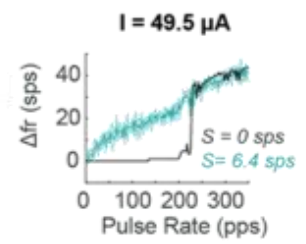

d

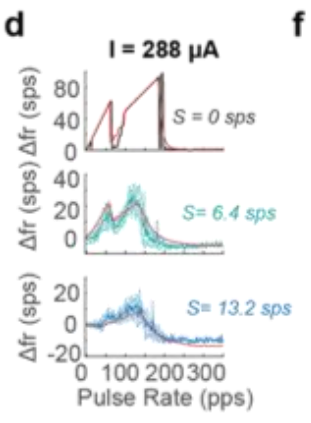

$\mathbf{f}$ e
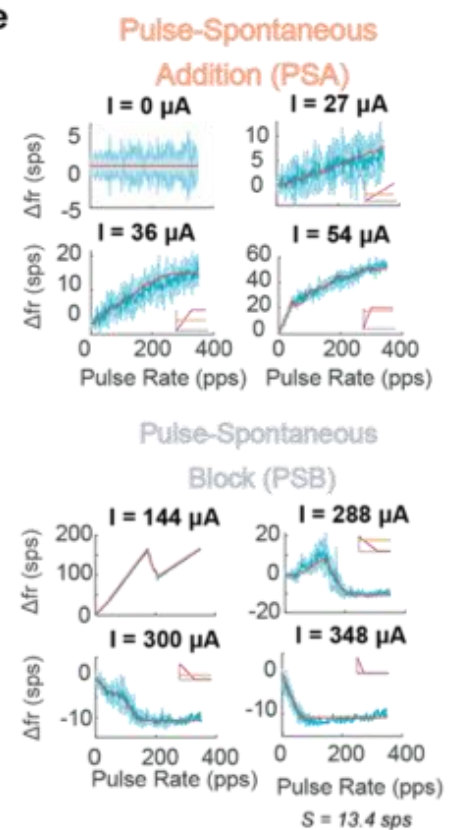

g Spontaneous Pulse-Block (SPB)

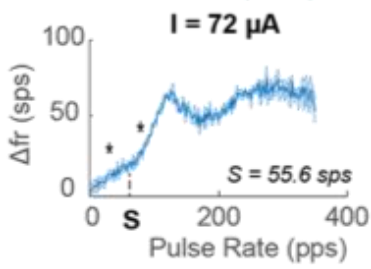

h

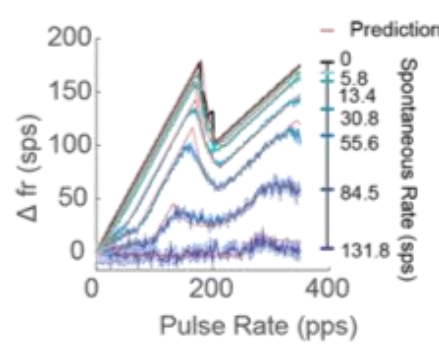

Figure 4. Interactions of Pulses with Spontaneous Firing. a. Interactions of spontaneous spikes with pulse-evoked activity at 30 pps and 80 pps. Spontaneous spikes are in green. b. At high and low amplitudes a pulse will interact with multiple spikes or a spike may interact with multiple pulses. Time of spontaneous activity compared to pulses takes an approximately uniform distribution between pulses, having equal chance of effecting all pulses (psp). As $\rho$ decreases, the after-pulse window affects a greater portion of spontaneous activity (pps), so pps( $\rho)$. As current increases both probability of pulses affecting spikes (PPs) and spikes affecting pulses (PsP) increase together. The probabilities fall between 0 to 1 . c. Presence of even a small amount of spontaneous activity (6.4 sps) leads to smoothing of pulse=induced effects leading to facilitation even at pulse rates below the rate required for typical pulse-pulse facilitation. $\mathbf{d}$. This also leads to reduction and eventually disappearance of PDL effects as $S$ increases to 13.2 sps. e. Facilitation effects can be explained with psp and pps increasing to 1 . High amplitude blocking can be explained with psp and pps returning to 0 . $\mathbf{h}$. The main spontaneous-pulse effect is blocking at mid-range $I$. The same pattern produced with $S=0$ is observed but with $S$ lower firing rate and smoothed out partial elimination zones. 
a

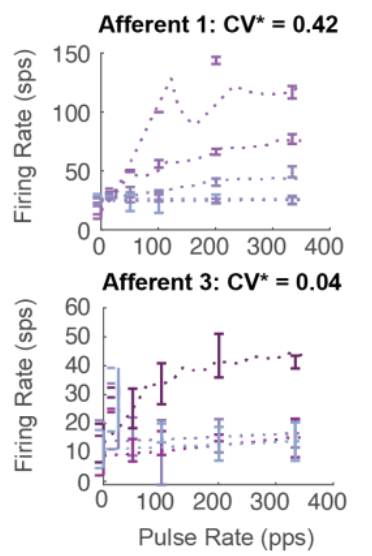

b

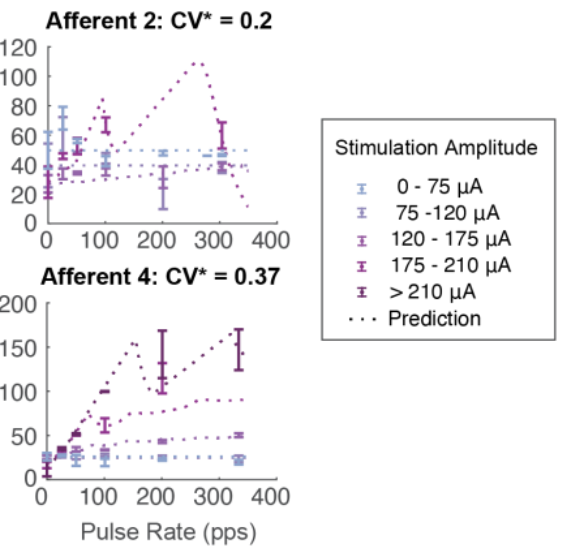

C

Effects of Spontaneous Rate
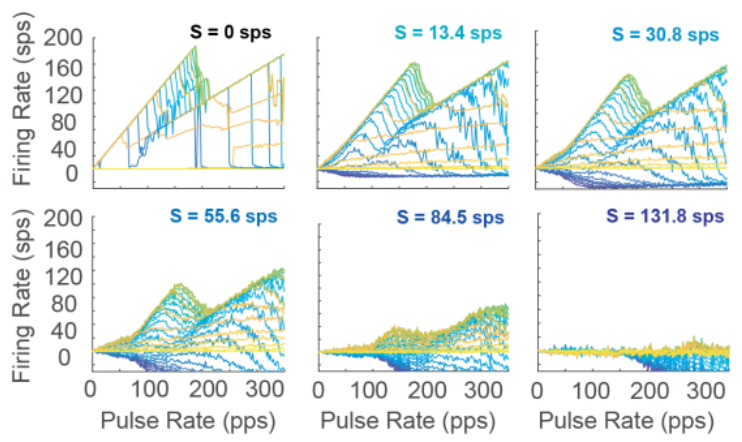

Pulse Amplitude ( $\mu \mathrm{A})$

0

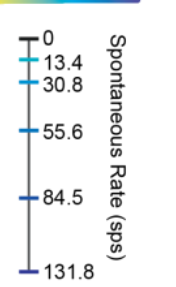

e

Pulse Timing Jitter
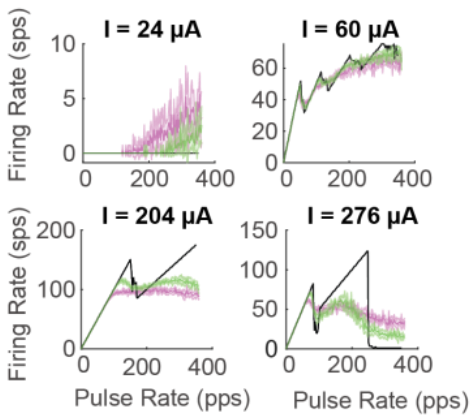

No Jitter

2 ms Jitter f
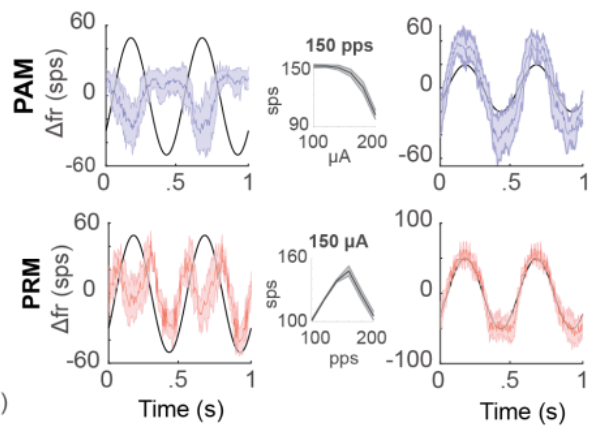

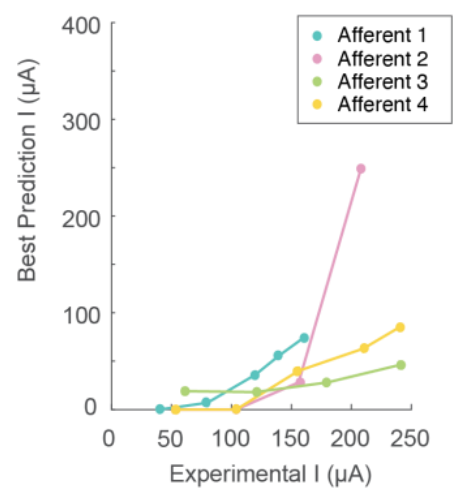

d

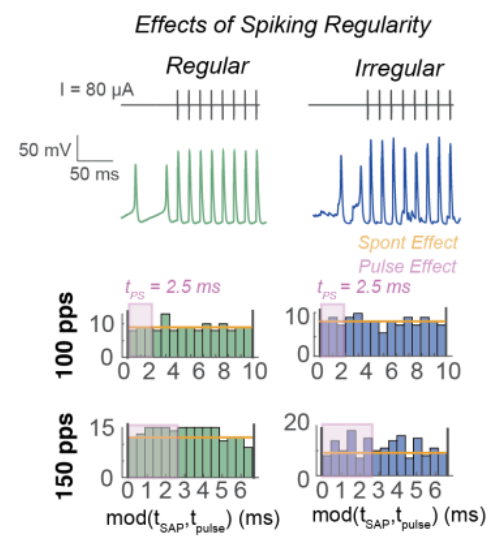

g

Behavioral Effect
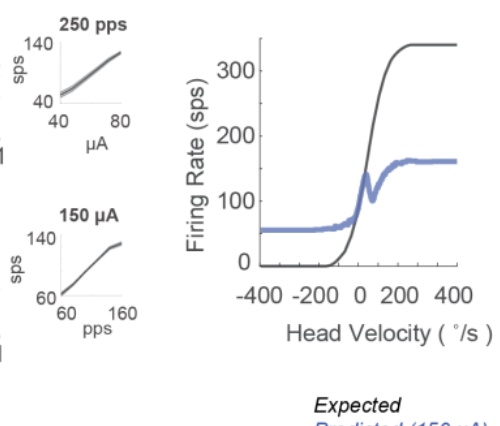

Figure 5. Prediction of Experimental Vestibular Afferent Responses. a. Four afferents varying from regular $\left(\mathrm{CV}^{*}=0.04\right)$ to irregular $\left(\mathrm{CV}^{*}=0.42\right)$ in response to pulsatile stimulation with 0 to $240 \mu \mathrm{A}$. Predictions are shown in matching color to the data (dashed). Predictions used the same parameterization with respect to $I$ and $S$. $S$ was chosen as the firing rate immediately prior to the pulse block. Only the value of I was chosen to best fit the experimental data. b. The relationship between $I_{\text {pred }}$, the $I$ of best fit and the experimental value delivered at the electrode for each afferent. c. Spontaneous activity has a massive effect on induced firing rate. The same currents (yellow-blue) lead to addition versus blocking depending on $S$. Each square shows the firing rate at the same amplitude with $S=0$ to $S=131 \mathrm{sps}$. d. Regularity does not significantly affect the probability of pulse-spontaneous activity interactions for $p r>S$. So, we can make the simplifying assumption that regularity does not have a large influence. e. Pulse delivery timing often occurs with a jitter. We tested the effect of a jitter of $1 \mathrm{~ms}$ (green) std and $2 \mathrm{~ms}$ std (purple) compared to pulses with no jitter (black). We see the jitter smooths the partial elimination zone significantly, similarly to spontaneous activity. $f$. Pulse rate modulation (PRM) and pulse amplitude modulation (PAM) with sinusoids was simulated on the model with modulation frequency of 1 $\mathrm{Hz}$. The predicted induced pulse rate for sinusoidal pulse rate (red) and pulse amplitude (blue) modulation compared to predicted changes (black). For given current range and fixed pulse rate or vice versa the prediction of our equations for change in firing rate.(bottom right). g. Firing rate of a normal vestibular afferent in response to head velocity (black) vs. predicted firing rate if the pulse train equivalent to natural firing rate was presented to the afferent (blue). 


\section{Methods}

\section{Vestibular Afferent Experimental Paradigm}

\section{Surgical procedures}

Chronic stimulation and recording experiments were performed in three male rhesus monkeys (ages 4, 5 and 7 years old). All procedures were approved by both the McGill University Animal Care Committee and the Johns Hopkins Animal Care and Use Committee, in addition to following the guidelines of the Canadian Council on Animal Care and the National Institutes of Health.

Surgeries were performed as described in Mitchell, Della Santina \& Cullen. ${ }^{48}$ An electrode array was implanted into the left labyrinth via transmastoid approach such that the strut of bone between the ampullae of the superior and horizontal semicircular canals was kept intact. This acted as a stop for the electrode array. Reference electrodes were inserted into the common crus of the labyrinth and extracranial musculature. The animals were given a 2-week recovery time from surgery before experiments were performed. Recorded electrodes were mapped to the electrodes that delivered the evoked highest activity.

\section{Data acquisition}

During a recording session monkeys were comfortably seated in a primate chair. An enamel-insulated tungsten microelectrode (8-10 M $\Omega$ impedance; Frederick Haer Co., Bowdoinham, ME) was used to record single unit activity of the vestibular nuclei neurons. Single unit activity was sampled at $40 \mathrm{kHz}$. An adaptive filter (Artifact Zapper, Riverbend Instruments) was used on-line to remove simulation artifacts in the neural recordings. If any residual artifact remained, we performed a template deletion offline in which an average waveform of the artifact was subtracted from the recording trace. Each unit was analyzed offline to ensure proper isolation. Subsequent analysis was performed using custom algorithms (Matlab, The MathWorks). After vestibular nuclei neurons and afferent fibers were characterized, all neuronal activity was recorded in complete darkness while the monkeys were head-fixed.

\section{Experimental Stimulation Paradigms}

Pulses were applied using an isolated pulse stimulator (A-M systems), which delivered biphasic pulses with 150 $\mu \mathrm{s}$ stimulation phases and no interphase gap. The same single unit stimulation paradigm used in the monkey physiology experiments was replicated in silico on the biophysical model. During each pulse block, we delivered $1 \mathrm{~s}$ pulse trains at rates from 25 to 300 pps (Fig. 1B). These rates were chosen because they fall within the physiological range of vestibular afferent firing ${ }^{22}$. Fixed-rate pulse blocks were delivered at a fixed pulse amplitude in order from lowest to highest pulse rate. There were typically four repetitions of each pulse rate block before increasing the pulse rate. For several there were only three repetitions. For most pulse blocks there was a $500 \mathrm{~ms}$ or $1 \mathrm{~s}$ window between blocks. The maximum current was set to $80 \%$ of the minimum value that caused visible facial muscle activation in response to pulses delivered at 300 pps. Pulse rate experiments were performed with a fixed current amplitude and repeated at amplitudes from $25 \%, 50 \%, 75 \%$, $87.5 \%$, and $100 \%$ of maximum amplitude (Fig. 1B).

\section{Data Analysis}

Our assay of neural responses is firing rate in response to blocks of pulses. Therefore, induced firing rate was measured as the number of APs that occurred from the onset of the first pulse in a pulse block to the offset of the last pulse in the block divided by the length of that time window. There were noticeable experimental differences in spontaneous activity before and after pulse blocks. Therefore, spontaneous activity was measured as the firing rate in the window preceding a pulse train, excluding the first $50 \mathrm{~ms}$, if the window 
occurs after another pulse block. This was done to avoid remaining effects from the previous pulse train. Many stimulation paradigms assume a linear relation between pulse rate and firing rate. To test this hypothesis, data from all repetitions of pulse rate blocks at a single current amplitude were fit with the line best fit with a $y$ intercept equal to the average spontaneous rate $(S): f r=m p r+S$. The slopes of best fit are compared to the unity line, the slope if each pulses produced one AP (Fig. 1C).

\section{Data Fitting with Equation $\mathrm{fr}=\phi(p r, l, S)$}

Simulations revealed predictable, smooth transitions between effects of pulses with the change in pulse amplitude and spontaneous rate. These effects were captured through an equation $f r=\phi(p r, I, S)$ (Eq.1) explained further below that takes measurable and controllable values as inputs: pulse rate $p r$, pulse amplitude $I$, and spontaneous rate $S$. The equation captures effects from facilitation to blocking at 0 to $350 \mu \mathrm{A}$ as fitted to a single irregular afferent simulation. Only the magnitude of parameters changes as a function of $I$ and $S$ to produce these effects (Fig. 2).

The experimental data were fit by using the measured values of $\mathrm{pr}$ and $\mathrm{S}$ and optimizing on predicted current, $I_{\text {pred. }} I_{\text {pred }}$ was chosen as the value that minimized the two-dimensional rms between the data at 25 to 300 pps and predictions. The two-dimensional error was taken between a prediction $\mathrm{fr}_{\text {pred }}=\phi\left([1: 300] p p s, I_{\text {pred }} S\right)$ and the 8 experimental values. The $(x, y)$ error for each of the 8 sampled pulse rates $(k)$ was taken as the minimum distance between the closest point on $f r_{p r e d}$ and the $k$ th pulse rate - firing combination $\left(\rho_{k}, f r_{k}\right)$ :

$$
r m s_{\rho f r}=\sum_{\rho=1}^{8} \min _{\rho}\left\{\sqrt{\left(\rho-\rho_{k}\right)^{2}+\left(\phi\left(p r, I_{\text {pred }}, S\right)-\overline{f r} r_{k}\right)^{2}}\right\}_{\rho=1}^{300}
$$

The total error for $I_{\text {pred }}$ was the sum of the error at each of the 8 points.

The only restrictions on $I_{\text {pred }}$ were the value that minimized the rms error described above and that the value was weighted by the size of $/$ pred:

$$
\epsilon=r m s_{\rho f r}+.5 \frac{I_{\text {pred }}}{200}
$$

The second rule was enforced because many pr-fr combinations are the same at high and low / values. Also, between 100-200 $\mu \mathrm{A}$, the relationship is approximately static. With the sparse sampling of pulse rate, this weighting helped assure $I_{\text {pred }}$ was not overestimated. Note, all model errors reported on the figure are standard rms measured at the pulse rates tested in the experimental data.

\section{Biophysical Modeling of Vestibular Afferents}

Vestibular afferents were simulated using a biophysical model that has been used previously by several groups including our own to study the effects of electrical stimulation on vestibular afferents. ${ }^{22-24}$ Past work from the lab showed this model can replicate experimental firing rates and changes in firing rate with pulsatile and direct current stimulation. ${ }^{22,24}$

We use an adapted version of the Hight and Kalluri model. More details can be found in Steinhardt and Fridman. ${ }^{24}$ In brief, Hight \& Kalluri showed that vestibular firing can be simulated accurately by assuming cells have the same shape and size ${ }^{23}$ Type I and Type II vestibular afferents ${ }^{49}$ are modeled as differing only in channel expression and EPSC magnitude $(K)$. Spontaneous rate can be set by changing the average inter-EPSC arrival interval $(\mathrm{m})$.

The membrane potential $(V)$ varies as:

$$
\frac{d V}{d t}=\frac{1}{\left(C_{m} S\right)\left(I_{N a}+I_{K L}+I_{K H}+I_{\text {leak }}+I_{\text {epsc }}+I_{\text {stim }}\right)}
$$


where in addition to the current from each channel, membrane potential is influenced by the EPSCs arriving at axon $\left(I_{\text {epsc }}\right)$ and the injected current $\left(I_{\text {stim }}\right)$. The system of equations in ${ }^{23}$ represents each cell as a single node with overall surface area, $S=1.1 \cdot 10^{-5} \mathrm{~cm}^{2}$ and capacitance $C_{m}=0.9 \mathrm{mF} / \mathrm{cm}^{2}$. Each channel is voltage-gated and dependent on a conductance, an open state and a closed state variable: $\mathrm{Na}\left(\mathrm{g}_{\mathrm{Na}}, \mathrm{m}, \mathrm{h}\right), \mathrm{KH}\left(\mathrm{g}_{\mathrm{KH}}, \mathrm{n}, \mathrm{p}\right), \mathrm{KL}$ $\left(g_{\mathrm{KL}}, \mathrm{w}, \mathrm{z}\right)$. We simulate the electrode at $2 \mathrm{~mm}$ from the simulated afferent which causes the firing threshold around $56 \mu \mathrm{A}$ for a typical neuron.

For this study, the simulations used to parameterize our equations were performed on a single model of an irregular vestibular afferent. We found that conductance values of $\mathrm{g}_{\mathrm{Na}}=13 \mathrm{mS} / \mathrm{cm}^{2}, \mathrm{~g}_{\mathrm{KH}}=2.8 \mathrm{mS} / \mathrm{cm}^{2}$, and $\mathrm{g}_{\mathrm{KL}}$ $=1 \mathrm{mS} / \mathrm{cm}^{2}$ and EPSCs with $\mathrm{K}=1$ and $\mathrm{m}=1.3 \mathrm{~ms}$ matched previously published experimental findings at pulse rates from 25 to 300 pps (Fig. 1e). We kept these conductance values for all irregular afferent simulations.

For studies with the effects of spontaneous rates on firing. The channel conductance values were kept the same but $m$ was set to $0.25,0.5,1,2,4$, and 8 . To model the axon with no spontaneous activity, we simply did not add EPSCs.

Additionally, we assessed the effect of firing regularity on induced firing rate. The irregular neuron ( $f r=$ 36.6 $\pm 0.9 \mathrm{sps}, \mathrm{CV}=0.57$, where $\mathrm{CV}$ is Coefficient of Variance), was modeled with $\mathrm{K}=1$, and $\mathrm{m}=1.65 \mathrm{~ms}$. A conductance matched regular neuron ( $f r=33.8 \pm 0.4 \mathrm{sps}, C V=0.09$ ) was also modeled with $\mathrm{g}_{\mathrm{Na}}=13 \mathrm{mS} / \mathrm{cm}^{2}$, $\mathrm{g}_{\mathrm{KH}}=2.8 \mathrm{mS} / \mathrm{cm}^{2}$, and $\mathrm{g}_{\mathrm{KL}}=0 \mathrm{mS} / \mathrm{cm}^{2}, \mathrm{~K}=0.025$, and $\mathrm{m}=0.09 \mathrm{~ms}$.

We tested the effects of conductance values on the pr-fr mapping with current amplitude. We used conductance values that produced firing rates similar to those observed in a previous in vitro experiment with and without exposure to DC current: ${ }^{24,50} \mathrm{~g}_{\mathrm{Na}}=7.8 \mathrm{mS} / \mathrm{cm}^{2}, \mathrm{~g}_{\mathrm{KH}}=11.2 \mathrm{mS} / \mathrm{cm}^{2}$, and $\mathrm{g}_{\mathrm{KL}}=1.1 \mathrm{mS} / \mathrm{cm}^{2}, \mathrm{~K}=1 . \mathrm{m}$ was again varied from 0.25 to $8 \mathrm{~ms}$.

We found no evidence of pulsatile stimulation affecting the hair cell, so all DC hair cell related effects (adaptation, the non-quantal effect, etc.) were not activate in these simulations. The simulation is run using the Euler method to update all variables through each of the channels.

\section{Simulated Pulsatile Stimulation Experiments}

The experiment conducted in monkeys was repeated in silico with finer sampling of current amplitudes and pulse rates. Electrodes were simulated as being placed $2 \mathrm{~mm}$ from the vestibular afferent axon. In addition to the pulse rates used experimentally, pulse rates from 1 to 300 pps in steps of 1 pps were delivered for 1 second. Five repetitions were performed for each current amplitude, spontaneous rate, and pulse rate combination. Pulse amplitude was varied from 0 to $360 \mu \mathrm{A}$ in steps of $12 \mu \mathrm{A}$ and used to parameterize equations values. We interpolated between these values to create a smooth function for predicting induced firing rates.

This combination of experiments was repeated on the irregular neuron, regular neuron, and low conduction/in vitro neuron. It was also repeated for all values of $\mu$ to map how these effects change with different levels of spontaneous activity. 


\section{Jitter Experiment}

To assess the effect of jittered pulse delivery time on induced firing rate, we performed the same simulation. Instead of delivering perfectly timed pulses we added a gaussian noise term with standard deviation of $1 \mathrm{~ms}$ or $2 \mathrm{~ms}$ to the exact pulse timing to simulate delay or advancement in the delivery of regularly scheduled pulses (Fig. 5e).

\section{Pulse Rate and Amplitude Modulation}

To test how these rules apply to sinusoidal modulation, as used in various prosthetic algorithms, PRM and PAM was simulated within a common range for vestibular prostheses. ${ }^{12,26,51}$ Pulse rates were modulated by steps of 20 or $50 \mathrm{pps} / \mu \mathrm{A}$ around values where non-linearities or linearities were observed in the one-second fixed pulse rate experiments (Fig. $5 f$ grey). Sinusoidal PRM (red) and PAM (blue) modulation was simulated for the same afferent with a 42 sps baseline firing rate (Fig. $5 f$ ).

\section{Predictive Equation}

The observed effects at the axon were transformed into equations that depended on measurable or controllable variables: pulse amplitude $(I)$ delivered from the electrode, pulse rate, as inter-pulse interval $(\rho)$, and spontaneous rate $(S)$. Equations were not optimized for real-time usage. The goal was to create equations that captured the observed effects and their mechanisms without dependence on time. The equations were made to reflect mechanism. Variables were shown to vary with $I$ and $S$. They were best fit to each I-S combination of the simulated afferent. All variables that were best fit are marked with " ' " in the following equations.

\section{Pulse-Pulse Interactions}

Pulse-Pulse Block/Partial Block (PPB)

The most substantial effect of pulses in the absence of EPSCs is pulses blocking following pulses from becoming APs. The visible refractory period after a pulse is driven by an underlying change in channel dynamics that leads to a zone where all pulses are blocked followed by a zone of exponentially decreasing blocking effects until pulses have zero probability of being blocked (Fig. 3a-c). Because the following pulses are blocked, the pulses within the block zone do not extend these effects, leading to the induced firing rate being a subharmonic of the pulse rate:

$$
f r_{P P}=\frac{\left(\frac{1}{\rho}\right)}{\operatorname{ceil}\left(\frac{t_{b}(I) \prime}{\rho}\right)+P E(I, \rho)}
$$

where the length of the full block zone $t_{b}(I)$ changes with $/$ (Fig. 2, 3b). $t_{b}$ is large when $/$ is small (due to channels driving the membrane towards resting state) and when $/$ is large (due to the pulses causing large changes that push channels into unnatural states). At I around 100-200 $\mu \mathrm{A}$ in silico, $t_{b}$ remains at approximately the same minimum values, as pulses are strong enough to consistently drive firing but not strong enough to cause extreme changes in channel states. The ratio of $\rho$ to $t_{b}$ determines what fraction of pulses are blocked, so the pulse rate at which $1 / 2$ or $1 / 3$ of pulses become APs is easily calculable as $\left(n / t_{b}\right) p p s$, where $n$ is the denominator of the fraction. This effect is captured with division by the ceil) term.

After $t_{b}$ until $t_{p b}(I)$, the subsequent pulse is not blocked with certainty, but the probability of being blocked decreases with time. We call this zone the partial elimination (PE) zone. $t_{p b}$ changes with / similarly to $t_{b}$ (Fig. $2 b-c)$. We implement PE with respect to the transition point between each bend $(n)$ at which the firing rate changes from $\mathrm{pr} / \mathrm{n}$ to $\mathrm{pr} /(n+1)$ due to the observed changing in length of PE with each transition or bend in the 
pulse rate-firing rate relationship. Because transitions happen at each $n / t_{b}$ pps pulse rate, the pulse rate at the start of PE for that transition can never be less than $(n-1) / t_{b}$. Therefore. we express the length of the PE zone as a fraction $p_{p b}^{\prime}$ times $t_{b}$ :

$$
t_{p b}(n)=\frac{t_{b}^{\prime}}{n-\min \left\{.99, p_{p b}^{\prime}\right\}}
$$

As $\rho$ decreases such that $n>1, p_{p b}^{\prime}$ becomes an increasingly larger (Fig. 3c-1). This is due to a change between $n$ and $n-1$ at large $n$ being equivalent to a smaller time between pulses. The length of $t_{p b}$ does decrease as $n$ increases.

PE is implemented such that between $\frac{t_{b}}{n}$ and $t_{p b}(n)$, the PE term takes values that linearly decrease from 1 to 0 as the pulse rate approaches $\frac{n}{t_{p b}}$. This term adds to the ceil) term so that the transition is not abrupt from fr = $\mathrm{pr} / \mathrm{n}$ to $\mathrm{fr}=\mathrm{pr} /(n+1)$ but instead has an exponential decay from one harmonic line to the next (Fig. $3 \mathrm{c} \mathrm{red})$. The strength of PE grows with $I$, which could be well characterized with the scaling $\kappa$ :

$$
\begin{aligned}
& \kappa=\left(I-\frac{\alpha^{\prime}(S)}{300}\right)^{4} \\
& P E(I, \rho)=\left\{\begin{array}{cl}
\sum_{n=1}^{N} \min \left\{1,(1+\kappa(I, S))\left(1-\frac{\rho-\frac{t_{b}^{\prime}}{n}}{\left(t_{p b}(n)-\frac{t_{b}^{\prime}}{n}\right)}\right)\right\}, & t_{p b}(n)<\rho<t_{b}^{\prime} / n \\
0 \quad, \quad \text { else }
\end{array}\right.
\end{aligned}
$$

At midrange I, the scaling effect with / causes lower prs to transition to the next subharmonic $(f r=p r /(n+1))$ but it never causes a harmonic to skip to over $n+1$. As I increases, such as at I $=192 \mu \mathrm{A}$, this leads to a sharper transition from one line to the next (Fig. 3c-2). This effect is implemented with the min term such that PE $£ 1$. When spontaneous rate was increased, we found that $\kappa$, the scaling up of $P E$ effects, occurs at a lower $I$ due to the spontaneous activity distorting and expanding the timing and likelihood of pulses causing large changes along the axon for smaller changes in membrane voltage. This effect is embedded in the equation through the $\alpha^{\prime}$ which increase with $S$.

At very high I ( $>204 \mu A$ in our mapping) suppression effects occurred that caused axonal firing to shut down in longer, unnatural dynamic loops. Two main effects at high amplitudes (Pulse Dynamic Loop (PDL) and Suppression of Future Pulses (SFP)) were implemented as variations of the $P E$ function.

\section{Pulse Dynamic Loop (PDL)}

At the transition from $n=1$ to $n=2$, with no spontaneous activity we see that the transition overshoots and comes returns to $n=2$ during the PE time (Fig. 3F1). We model this with a similar function with reverse directionality of exponential decay:

$$
\begin{aligned}
& \text { for } t_{p b}(1)<\rho<t_{b}^{\prime} \\
& \qquad \mathrm{PE}_{1}=\operatorname{ceil}\left(\kappa(I, S)\left(\frac{\rho-t_{b}^{\prime}}{\left(t_{p b}(1)-t_{b}^{\prime}\right)}\right)\right)
\end{aligned}
$$

Suppression of Future Pulses (SFP)

Additionally, instead of the pulses within the immediate refractory period being blocked, the delivery of an additional pulse pushes axonal dynamics to a state of full suppression after $n=2$. We again see this transition is an exponential decay to $f r=0$ as opposed to a sharp drop (Fig. 3F2). This rule is implemented as: 
for $\rho>=t_{p b}(2)$,

$$
P E_{2}=\left(200 / \beta^{\prime}(\mathrm{S})\right)\left(1-\frac{\rho-\frac{t_{b}^{\prime}}{2}}{\left(t_{p b}(2)^{\prime}-\frac{t_{b}^{\prime}}{2}\right)}\right)^{3}
$$

When spontaneous activity is included, we see that, with larger spontaneous activity, the slope of this decay is exceedingly slower. This effect is enforced by $\beta^{\prime}(S)$, which increases from 1 to 250 , when $S=130$ sps.

With these terms only, the induced firing rate simplifies to:

$$
f r_{P P}=\frac{\left(\frac{1}{\rho}\right)}{\left.\min \left\{2, \operatorname{ceill}\left(\frac{\left.t_{b}()^{\prime}\right)}{\rho}\right)\right\}+P E_{-} 1+P E_{-} 2\right)}
$$

The min term assures smooth transition to $f r=0$ without the bends that would typically occur at pulse rates that are multiples of $1 / t_{b}^{\prime}$.

\section{Pulse-Spontaneous Interactions}

Pulse-Spontaneous Additive (PSA), Pulse-Spontaneous Block (PSB), and Spontaneous-Pulse Block (SPB)

Regularly timed pulses break up time into windows of length $\rho$ in which relative affects of pulses on spontaneous activity and vice versa can be approximated. We numerically implement this effect with $\bmod \left(t_{s,} \rho\right)$. This shows that spontaneous spikes are distributed uniformly between 0 and $\rho$ ms after a pulse. So, pulses affect some fraction of the evenly distributed spontaneous activity, and all pulses are affected to some level by the ongoing spontaneous activity. As pr increases, the time between pulses is smaller but the probability of a spontaneous AP is equally likely, so a greater portion of spontaneous activity is affected per pulses. We call the probability of spontaneous activity affected by pulses, $p_{P S}$. The probability of pulses being affected by spontaneous activity and producing APs is $p_{S P}$. A simple approximation of these interactions would be:

$$
p_{P S}=\min \left\{1, \frac{\rho-t_{P S}(I, S)}{\rho}\right\}
$$

Where $t_{P S}$ is the time after a pulse when spontaneous APs are blocked.

$$
p_{S P}=\min \left\{1, \frac{\left(t_{S P} S\right)}{T}\right\}
$$

Where $t_{S P}$ is the time after a spontaneous AP when pulses are blocked, and $T$ is the total length of the time window. $t_{S P}$ and therefore $p_{S P}$ only depends on $I$, as spontaneous rate will remain the same for a neuron. With this approximation, $t_{P S}$ will only depend on $I$ and $p_{P S}$ will increase linearly with the pulse rate until reaching 1 . To simplify fitting, we therefore fit $p_{P S}^{\prime}$ and $p_{S P}^{\prime}$ directly depending on $I$. Spontaneous rate did not have large effects on these variables. Implementation would likely not be largely affected if these variables were kept the same for any spontaneous rate.

These pulse-spontaneous interactions took two forms. At low amplitudes, they worked together to produce facilitation of pulses at amplitudes that a silent axon could not produce activity (Fig. 3). By I $=54 \mu \mathrm{A}$, both probabilities reach 1 , representing the transition from pulses facilitating with spontaneous activity to blocking interactions (Fig. 3b,e). At very high $I$, the same equations taking the same shape can be used to describe pulses blocking spontaneous activity and spontaneous activity blocking pulses (Fig. 3f). These spontaneous activity effects are linked to EPSC facilitating or being facilitated by pulses into becoming APs, or pulses becoming large enough that underlying EPSC activity can quickly transition the axon to suppressed state. fit $p_{P S}^{\prime}$ and $p_{S P}^{\prime}$ both increases to 1 sigmoidally around $50 \mu \mathrm{A}$. The blocking effects of pulses on spontaneous activity start around $156 \mu \mathrm{A}$ and are significantly dependent on spontaneous activity. The spontaneous activity scaling down pulse-induced firing changes rapidly around $290 \mu \mathrm{A}$ without strong dependence on $S$. 


\section{Spontaneous-Pulse Full Block Effects}

Between about 50 and $290 \mu \mathrm{A}$, spontaneous activity blocks pulses to a more limited degree. These effects are likely due to spontaneous APs and not EPSCs, as the quantities directly relate to $S$. The largest effect is that, as $S$ increases, the same pr-fr relationships occur as in silent neurons but reduced by $S$ (Fig. $4 \mathrm{~g}$-h). Voltage traces indicate this is due to spontaneous activity blocking pulses, and pulses are only shown to block spontaneous activity at significantly larger amplitudes. Thus, $\mathrm{S}$ is subtracted from $f r_{P}$. Additionally, there is a bend in the PFR (Fig. 3g). As described above, until $\rho=t_{b}(I)$, the slope of the PFR should be 1. Prior to this bend, the slope is significantly lower, and afterwards it is 1 . We find that pulses are relatively weak, such that below approximately $P R=S$, pulses are often blocked by spontaneous APs, while, for $P R>S$ pulses seem to alter dynamics so that multiple pulses survive between spontaneous APs (Fig. 4g). We fit this non-linearity as an additionally slope until $S$ that increases with $I$ and is scaled down with $S(\delta)$.

Facilitation ends around $50 \mu \mathrm{A}$, but this threshold current varies with $S$, so we call it $\mathrm{I}_{\mathrm{fac}}(S)$ and include facilitation as:

For $I<\mathrm{I}_{\text {fac }}(\mathrm{S})$,

$$
\begin{aligned}
f r_{P} & =p_{P S}^{\prime} P R, & & P R<\left(0.8 / p_{S P}{ }^{\prime}\right) \\
& =p_{S P}^{\prime} f r_{P P}, & & P R>\left(0.8 / p_{P S}{ }^{\prime}\right)
\end{aligned}
$$

For $I>\mathrm{I}_{\text {fac }}(S)$,

$$
\begin{array}{rlr}
f r_{P} & =\delta^{\prime}(I, S) P R, & P R<S \\
& =p_{S P}^{\prime} f r_{P P}-S-f r_{P P}(S)+\delta^{\prime}(I, S) S, & P R>S \\
f r_{S} & =S-p_{P S}{ }^{\prime} P R, \quad I>156 \mu \mathrm{A} & \\
& =S \quad \text { else } \\
f r & =\max \left\{0, f r_{S}\right\}+\max \left\{0, f r_{P}\right\} &
\end{array}
$$

The max term assures each term does not go negative if blocking effects exceed $S$ or $p r$. 


\section{References}

1. Loeb, G. E. Neural Prosthetics:A Review of Empirical vs. Systems Engineering Strategies. vol. 2018 (2018).

2. Urdaneta, M. E., Kunigk, N. G., Delgado, F., Fried, S. I. \& Otto, K. J. Layer-specific parameters of intracortical microstimulation of the somatosensory cortex. Journal of Neural Engineering 18, (2021).

3. Keller, C. J. et al. Mapping human brain networks withcortico-ortical evoked potentials. Philosophical Transactions of the Royal Society B: Biological Sciences vol. 369 (2014).

4. Piccolino, M. Animal electricity and the birth of electrophysiology: the legacy of Luigi Galvani. Brain research bulletin 46, 381-407 (1998).

5. Piccolino, M. \& Bresadola, M. Shocking frogs. Galvani, Volta, and the electric origins of neuroscience. (2013).

6. Vincent, M. et al. The difference between electrical microstimulation and direct electrical stimulationtowards new opportunities for innovative functional brain mapping? Rev. Neurosci 27, 231-258 (2016).

7. Steinhardt, C. R. et al. Characterizing and predicting cortical evoked responses to direct electrical stimulation of the human brain. Brain Stimulation 13, 1218-1225 (2020).

8. Merrill, D. R., Bikson, M. \& Jefferys, J. G. R. Electrical stimulation of excitable tissue: design of efficacious and safe protocols. Journal of Neuroscience Methods 141, 171-198 (2005).

9. Khairuddin, S. et al. A Decade of Progress in Deep Brain Stimulation of the Subcallosal Cingulate for the Treatment of Depression. Journal of Clinical Medicine 9, 3260 (2020).

10. Cosetti, M. K. \& Waltzman, S. B. Cochlear implants: current status and future potential. http://dx.doi.org/10.1586/erd.11.12 8, 389-401 (2014).

11. Nowik, K., Langwińska-Wośko, E., Skopiński, P., Nowik, K. E. \& Szaflik, J. P. Bionic eye review - An update. Journal of Clinical Neuroscience vol. 78 8-19 (2020).

12. Boutros, P. J. et al. Continuous vestibular implant stimulation partially restores eye-stabilizing reflexes. JCI Insight 4, (2019).

13. Kolaya, E. \& Firestein, B. L. Deep Brain Stimulation: Challenges at the $<\mathrm{scp}>$ Tissue-Electrode $</ \mathrm{scp}>$ Interface and Current Solutions. Biotechnology Progress (2021) doi:10.1002/btpr.3179.

14. Zeng, F. G. Challenges in improving cochlear implant performance and accessibility. IEEE Transactions on Biomedical Engineering 64, 1662-1664 (2017).

15. Wei, C. G., Cao, K. \& Zeng, F. G. Mandarin tone recognition in cochlear-implant subjects. Hearing Research 197, 87-95 (2004).

16. F, H., M, G. \& E, J. Noise Reduction in Cochlear Implant Signal Processing: A Review and Recent Developments. IEEE reviews in biomedical engineering PP, 1-1 (2021).

17. EL, F. Advances in understanding the mechanisms of retinal degenerations. Clinical \& experimental optometry 103, 723-732 (2020).

18. Mitchell, D. E., della Santina, C. C. \& Cullen, K. E. Plasticity within non-cerebellar pathways rapidly shapes motor performance in vivo. Nature Communications 2016 7:1 7, 1-13 (2016).

19. Histed, M. H., Bonin, V. \& Reid, R. C. Direct Activation of Sparse, Distributed Populations of Cortical Neurons by Electrical Microstimulation. Neuron 63, 508-522 (2009).

20. Boulet, J., White, M. \& Bruce, I. C. Temporal Considerations for Stimulating Spiral Ganglion Neurons with Cochlear Implants. JARO - Journal of the Association for Research in Otolaryngology vol. 17 1-17 (2016). 
21. Hey, M., Müller-Deile, J., Hessel, H. \& Killian, M. Facilitation and refractoriness of the electrically evoked compound action potential. Hearing Research 355, 14-22 (2017).

22. Steinhardt, C. R. \& Fridman, G. Y. Predicting Response of Spontaneously Firing Afferents to Prosthetic Pulsatile Stimulation. in Proceedings of the Annual International Conference of the IEEE Engineering in Medicine and Biology Society, EMBS vols. 2020-July 2929-2933 (Institute of Electrical and Electronics Engineers Inc., 2020).

23. Hight, A. E. \& Kalluri, R. A biophysical model examining the role of low-voltage-activated potassium currents in shaping the responses of vestibular ganglion neurons. J Neurophysiol 116, 503-521 (2016).

24. Steinhardt, C. R. \& Fridman, G. Y. Direct current effects on afferent and hair cell to elicit natural firing patterns. iScience 24, 102205 (2021).

25. della Santina, C. C., Migliaccio, A. A. \& Patel, A. H. A Multichannel Semicircular Canal Neural Prosthesis Using Electrical Stimulation to Restore 3-D Vestibular Sensation. IEEE Transactions on Biomedical Engineering 54, 1016-1030 (2007).

26. Golub, J. S. et al. Prosthetic implantation of the human vestibular system. Otology and Neurotology 35 , 136-147 (2014).

27. Babalian, A. L., Ryugo, D. K. \& Rouiller, E. M. Discharge properties of identified cochlear nucleus neurons and auditory nerve fibers in response to repetitive electrical stimulation of the auditory nerve. Exp Brain Res 153, 452-460 (2003).

28. Litvak, L. M., Delgutte, B. \& Eddington, D. K. Improved temporal coding of sinusoids in electric stimulation of the auditory nerve using desynchronizing pulse trains. The Journal of the Acoustical Society of America 114, 2079-2098 (2003).

29. Bhadra, N. \& Kilgore, K. L. High-frequency electrical conduction block of mammalian peripheral motor nerve. Muscle and Nerve 32, 782-790 (2005).

30. Shepherd, R. K. \& Javel, E. Electrical stimulation of the auditory nerve: II. Effect of stimulus waveshape on single fibre response properties. Hearing Research 130, 171-188 (1999).

31. Mitchell, D. E., della Santina, C. C. \& Cullen, K. E. Plasticity within non-cerebellar pathways rapidly shapes motor performance in vivo. Nature Communications 7, 11238 (2016).

32. Wang, Z., Feng, Z. \& Wei, X. Axonal Stimulations With a Higher Frequency Generate More Randomness in Neuronal Firing Rather Than Increase Firing Rates in Rat Hippocampus. Frontiers in Neuroscience 12, 783 (2018).

33. GY, F., HT, B., AP, B. \& JW, J. Perceived intensity of somatosensory cortical electrical stimulation. Experimental brain research 203, 499-515 (2010).

34. Fries, P. Rhythms for Cognition: Communication through Coherence. Neuron 88, 220-235 (2015).

35. P, F. Rhythms for Cognition: Communication through Coherence. Neuron 88, 220-235 (2015).

36. Sun, D. Q. et al. Histopathologic Changes of the Inner ear in Rhesus Monkeys After Intratympanic Gentamicin Injection and Vestibular Prosthesis Electrode Array Implantation. Journal of the Association for Research in Otolaryngology: JARO 16, 373-87 (2015).

37. Carey, J. P. et al. Changes in the three-dimensional angular vestibulo-ocular reflex following intratympanic gentamicin for Ménière's disease. JARO - Journal of the Association for Research in Otolaryngology 3, 430-443 (2002).

38. Steinhardt, C. R. \& Fridman, G. Y. Direct current effects on afferent and hair cell to elicit natural firing patterns. iScience 24, 102205 (2021).

39. Aplin, F. P. \& Fridman, G. Y. Implantable Direct Current Neural Modulation: Theory, Feasibility, and Efficacy. Frontiers in Neuroscience vol. 13379 (2019). 
40. Aplin, F. P., Singh, D., Santina, C. C. della \& Fridman, G. Y. Combined ionic direct current and pulse frequency modulation improves the dynamic range of vestibular canal stimulation. Journal of Vestibular Research 1-8 (2019) doi:10.3233/ves-190651.

41. Aplin, F. P., Singh, D., Santina, C. C. della \& Fridman, G. Y. Ionic Direct Current Modulation for Combined Inhibition/Excitation of the Vestibular System. IEEE transactions on bio-medical engineering 66, 775-783 (2019).

42. Fridman, G. Y. \& della Santina, C. C. Safe direct current stimulator 2: concept and design. Conference proceedings: ... Annual International Conference of the IEEE Engineering in Medicine and Biology Society. IEEE Engineering in Medicine and Biology Society. Annual Conference 2013, 3126-3129 (2013).

43. Fridman, G. Y. \& della Santina, C. C. Safe Direct Current Stimulation to Expand Capabilities of Neural Prostheses. IEEE Transactions on Neural Systems and Rehabilitation Engineering 21, 319-328 (2013).

44. Mitchell, Di. E., della Santina, C. C. \& Cullen, K. E. Plasticity within excitatory and inhibitory pathways of the vestibulo-spinal circuitry guides changes in motor performance. Scientific Reports 7, 853 (2017).

45. Eatock, R. A. \& Songer, J. E. Vestibular Hair Cells and Afferents: Two Channels for Head Motion Signals. Annual Review of Neuroscience 34, 501-534 (2011).

46. Manca, M., Glowatzki, E., Roberts, D. C., Fridman, G. Y. \& Aplin, F. P. lonic direct current modulation evokes spike-rate adaptation in the vestibular periphery. Scientific reports 9, 18924 (2019).

47. Fridman, G. Y. \& della SAntina, C. C. Progress Toward Development of a Multichannel Vestibular Prosthesis for Treatment of Bilateral Vestibular Deficiency. The Anatomical Record: Advances in Integrative Anatomy and Evolutionary Biology 295, 2010-2029 (2012).

48. Davidovics, N. S., Fridman, G. Y., Chiang, B. \& della Santina, C. C. Effects of biphasic current pulse frequency, amplitude, duration, and interphase gap on eye movement responses to prosthetic electrical stimulation of the vestibular nerve. IEEE transactions on neural systems and rehabilitation engineering: $a$ publication of the IEEE Engineering in Medicine and Biology Society 19, 84-94 (2011). 
bioRxiv preprint doi: https://doi.org/10.1101/2021.08.18.456731; this version posted August 19, 2021. The copyright holder for this preprint (which was not certified by peer review) is the author/funder, who has granted bioRxiv a license to display the preprint in perpetuity. It is made available under aCC-BY-NC-ND 4.0 International license.

\section{Supplemental Figures}
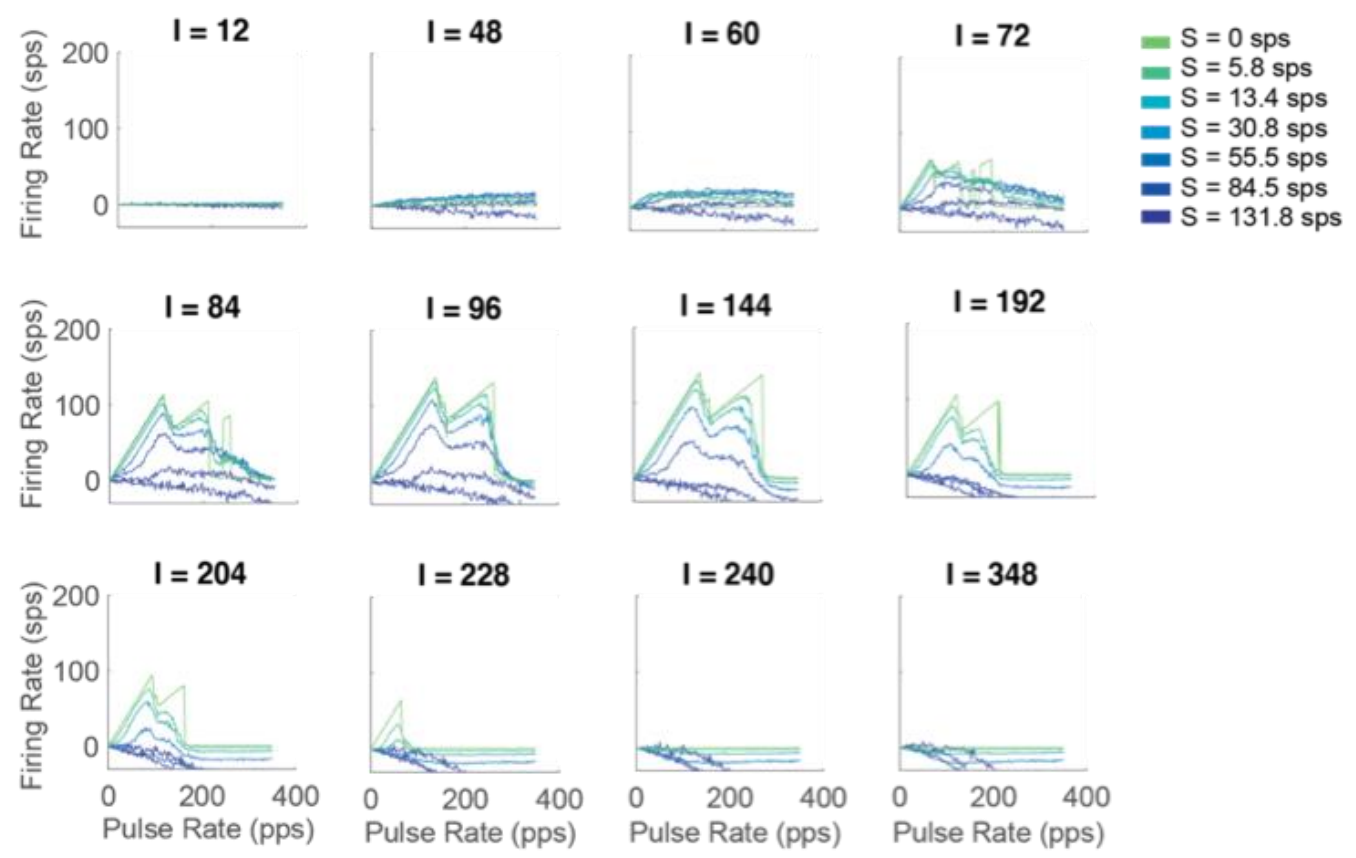

Supplemental Figure 1. Effect of Conductance on Response with Current. Mapping for pulse rate and firing rate relationship for same range of baseline spontaneous rates 0 to $131 \mathrm{sps}$. We note a quick transition from facilitation to pulsespontaneous blocking at lower current amplitudes. 

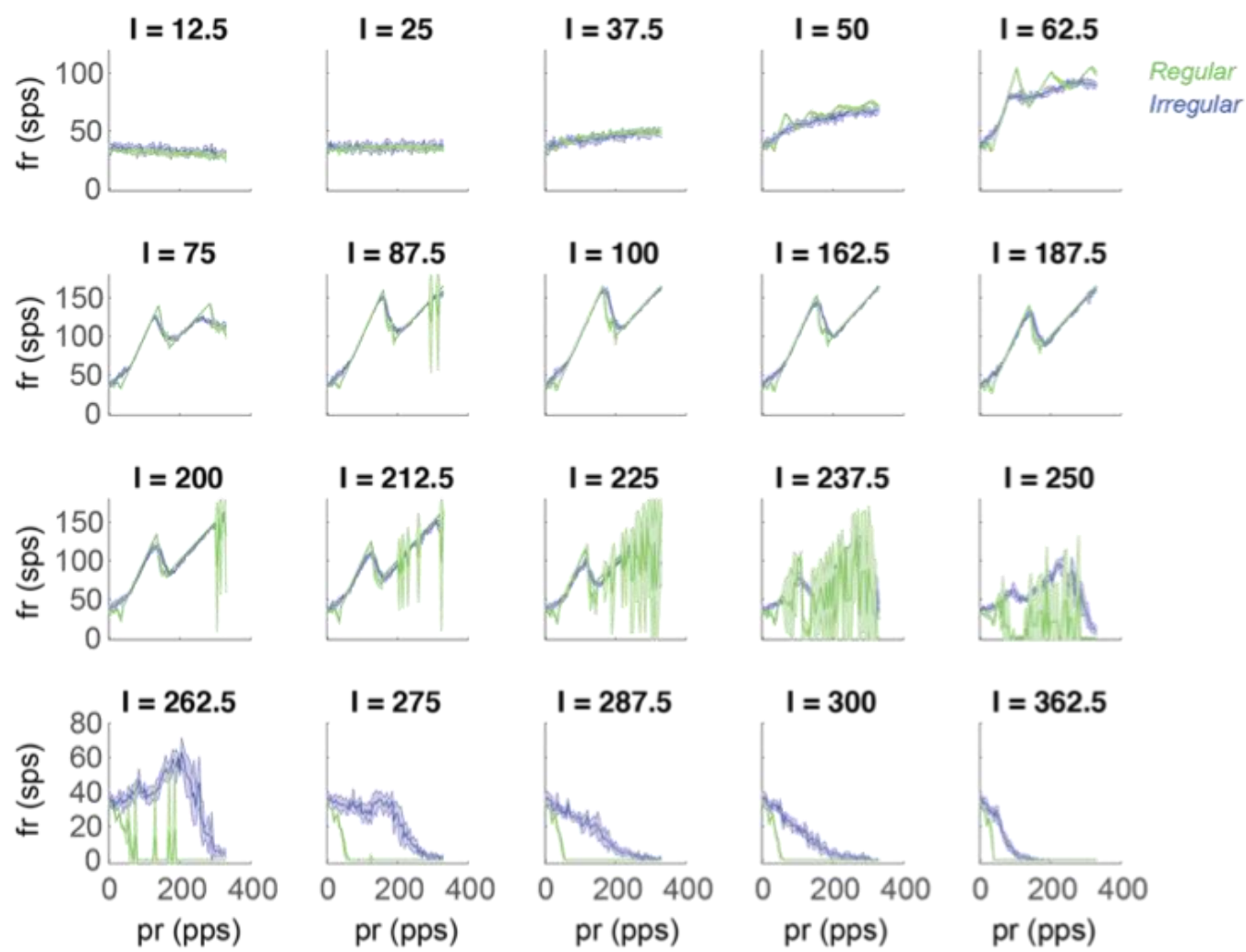

Supplemental Figure 2. Effect of Regularity on Pulsatile Stimulation. Mapping of pr-fr relationship for I from $0-362.5 \mu \mathrm{A}$ for regular (green, $\mathrm{CV}=0.09$ ) and irregular (blue, $\mathrm{CV}=0.57$ ) neuron with similar firing rate (32-36 sps). The response at the sampe current amplitude is plot for 5 repetitions, showing differences in the variance at different amplitude and the magnitude of the reduced length of the $P E$ zone for regular neurons. The start of blocking is observed to start at lower / for regular neurons. Additionally, when $p r<S$ the response dips, indicating a stronger SPB effect.

Supplemental Table 1. RMS across simulations at different spontaneous rates of normal conductance vestibular afferent. Error is reported as standard deviation. Mean is taken across 10 repetitions.

\begin{tabular}{|l|l|}
\hline Spontaneous rate (sps) & Average RMS (sps) \\
\hline 0 & $4.17 \pm 4.70$ \\
\hline 7.1 & $3.92 \pm 3.54$ \\
\hline 12.9 & $3.34 \pm 2.40$ \\
\hline 29.4 & $3.58 \pm 2.22$ \\
\hline 55.7 & $4.23 \pm 2.39$ \\
\hline 85.8 & $4.42 \pm 1.65$ \\
\hline 130.2 & $4.38 \pm 1.43$ \\
\hline
\end{tabular}

